\title{
ON THE BARTH-VAN DE VEN-TYURIN-SATO THEOREM
}

\author{
IVAN PENKOV AND ALEXANDER S. TIKHOMIROV
}

\begin{abstract}
The Barth-Van de Ven-Tyurin-Sato Theorem claims that any finite rank vector bundle on the infinite complex projective space $\mathbf{P}^{\infty}$ is isomorphic to a direct sum of line bundles. We establish sufficient conditions on a locally complete linear ind-variety $\mathbf{X}$ which ensure that the same result holds on $\mathbf{X}$. We then exhibit natural classes of locally complete linear ind-varieties which satisfy these sufficient conditions.
\end{abstract}

2000 Mathematics Subject Classification: Primary 14M15; Secondary 14J60, 32L05.

Keywords: ind-variety, vector bundle.

\section{INTRODUCTION}

The Barth-Van de Ven-Tyurin-Sato Theorem claims that any finite rank vector bundle on the infinite complex projective space $\mathbf{P}^{\infty}$ is isomorphic to a direct sum of line bundles. For rank-two bundles this was established by Barth and Van de Ven in [BV], and for finite rank bundles it was proved by Tyurin in [T] and Sato in [S1]. This topic was revived in the more recent papers [DP], [PT1], [PT2], where in particular the case of twisted ind-varieties was considered.

In the current paper we consider ind-varieties $\mathbf{X}=\lim _{\longrightarrow} X_{m}$ given by chains of embeddings of smooth complete algebraic varieties

$$
X_{1} \stackrel{\varphi_{1}}{\longrightarrow} X_{2} \stackrel{\varphi_{2}}{\longrightarrow} \cdots \stackrel{\varphi_{m-1}}{\longrightarrow} X_{m} \stackrel{\varphi_{m}}{\longrightarrow} \ldots
$$

We call such ind-varieties locally complete. A locally complete ind-variety $\mathbf{X}=\lim _{\longrightarrow} X_{m}$ is linear if the map on Picard groups induced by $\varphi_{i}$ is a surjection for almost all $i$. Our main objective is to give a reasonably general sufficient condition for the Barth-Van de Ven-Tyurin-Sato Theorem to hold on a locally complete ind-variety $\mathbf{X}$.

In the linear case, besides the results from the 1970-ies and the important results of Sato [S2],[S3] in which he considers a case when the Barth-Van de Ven-Tyurin-Sato Theorem no longer holds, some more recent results belong to Donin and Penkov [DP]. In particular, it is shown in [DP] that the Barth-Van de Ven-Tyurin-Sato Theorem holds on any linear direct limit $\mathbf{G}(\infty)=\lim G\left(k_{m}, \mathbb{C}^{n_{m}}\right)$, where $G\left(k_{m}, \mathbb{C}^{n_{m}}\right)$ denotes the grassmannian of $k_{m}$-dimensional subspaces in $\overrightarrow{\mathbb{C}^{m}}$, under the assumption that $\lim _{m \rightarrow \infty} k_{m}=\lim _{m \rightarrow \infty}\left(n_{m}-k_{m}\right)=\infty$. It turns out that there is a single isomorphism class of such ind-varieties. Nevertheless, there are other natural homogeneous ind-varieties on which the Barth-Van de Ven-Tyurin-Sato Theorem holds but which have not been considered in the literature. This applies in particular to linear direct limits of isotropic (orthogonal or symplectic) grassmannians, as well as to direct products of such direct limits.

For this reason we formulate a set of abstract conditions on a linear locally complete indvariety $\mathbf{X}$ which ensure that the Barth-Van de Ven-Tyurin-Sato Theorem (shortly, BVTS Theorem) holds. We then give many examples of ind-varieties $\mathbf{X}$ satisfying these sufficient conditions. An interesting new class of such ind-varieties consists of direct limits $\mathbf{Y}$ of linear sections $Y_{m}$ of $G\left(k_{m}, \mathbb{C}^{n_{m}}\right)$, where $\lim G\left(k_{m}, \mathbb{C}^{n_{m}}\right)=\mathbf{G}(\infty)$. Another class of ind-varieties on which the Barth-Van de Ven-Tyurin-Sato Theorem holds are certain ind-varieties of generalized flags, see subsection 6.3. 
Probably, there are more general sufficient conditions for the Barth-Van de Ven-Tyurin-Sato Theorem to hold on locally complete ind-varieties. In addition, for non-linear locally complete ind-varieties nothing seems to be known beyond the results of [PT2]. Therefore, providing a sufficient condition for the Barth-Van de Ven-Tyurin-Sato Theorem to hold on general locally complete ind-varieties remains a project for the future.

Acknowledgements. We acknowledge the support and hospitality of the Max Planck Institute for Mathematics in Bonn where the present paper was conceived. We also acknowledge partial support from the DFG through Priority Program "Representation Theory" (SPP 1388) at Jacobs University Bremen. A.S.T. has been financially supported by the Ministry of Education and Science of the Russian Federation.

\section{Linear ind-VARIETIES. Statement of the MAin RESUlt}

The ground field is $\mathbb{C}$. We use the term algebraic variety as a synonym for a reduced Noetherian scheme. If $E$ is a vector bundle (or simply a vector space), $E^{*}$ stands for the dual bundle (or dual space). We use the standard notation $\mathcal{O}_{\mathbb{P}^{n}}(a)$ for the line bundle $\mathcal{O}_{\mathbb{P}^{n}}(-1)^{\otimes-a}$, where $\mathcal{O}_{\mathbb{P}^{n}}(-1)$ is the tautological bundle on the complex $n$-dimensional projective space $\mathbb{P}^{n}$.

Recall that an ind-variety is the direct limit $\mathbf{X}=\underset{\longrightarrow}{\lim } X_{m}$ of a chain of morphisms of algebraic varieties

$$
X_{1} \stackrel{\varphi_{1}}{\rightarrow} X_{2} \stackrel{\varphi_{2}}{\rightarrow} \cdots \stackrel{\varphi_{m}-1}{\rightarrow} X_{m} \stackrel{\varphi_{m}}{\rightarrow} X_{m+1} \stackrel{\varphi_{m+1}}{\rightarrow} \ldots .
$$

Note that the direct limit of the chain (11) does not change if we replace the sequence $\left\{X_{m}\right\}_{m \geq 1}$ by a subsequence $\left\{X_{i_{m}}\right\}_{m \geq 1}$, and the morphisms $\varphi_{m}$ by the compositions $\tilde{\varphi}_{i_{m}}:=\varphi_{i_{m+1}-1} \circ \ldots \circ$ $\varphi_{i_{m}+1} \circ \varphi_{i_{m}}$.

Let $\mathbf{X}$ be the direct limit of (1) and $\mathbf{X}^{\prime}$ be the direct limit of a chain

$$
X_{1}^{\prime} \stackrel{\varphi_{1}^{\prime}}{\rightarrow} X_{2}^{\prime} \stackrel{\varphi_{2}^{\prime}}{\rightarrow} \ldots \stackrel{\varphi_{m-1}^{\prime}}{\rightarrow} X_{m}^{\prime} \stackrel{\varphi_{m}^{\prime}}{\rightarrow} X_{m+1}^{\prime} \stackrel{\varphi_{m+1}^{\prime}}{\rightarrow} \ldots .
$$

A morphism of ind-varieties $\mathbf{f}: \mathbf{X} \rightarrow \mathbf{X}^{\prime}$ is a map from $\mathbf{X}$ to $\mathbf{X}^{\prime}$ induced by a collection of morphisms of algebraic varieties $\left\{f_{m}: X_{m} \rightarrow Y_{n_{m}}\right\}_{m \geq 1}$ such that $\psi_{n_{m}} \circ f_{m}=f_{m+1} \circ \varphi_{m}$ for all $m \geq 1$. The identity morphism $\mathrm{id}_{\mathbf{X}}$ is a morphism which coincides with the identity as a set-theoretic map from $\mathbf{X}$ to $\mathbf{X}$. A morphism $\mathbf{f}: \mathbf{X} \rightarrow \mathbf{X}^{\prime}$ is an isomorphism if there exists a morphism $\mathbf{g}: \mathbf{X}^{\prime} \rightarrow \mathbf{X}$ such that $\mathbf{g} \circ \mathbf{f}=\mathrm{id}_{\mathbf{X}}$ and $\mathbf{f} \circ \mathbf{g}=\mathrm{id}_{\mathbf{X}^{\prime}}$.

In what follows we only consider chains (1) such that $X_{m}$ are complete algebraic varieties, $\lim _{m \rightarrow \infty}\left(\operatorname{dim} X_{m}\right)=\infty$, and the morphisms $\varphi_{m}$ are embeddings. We call such ind-varieties locally complete. Furthermore, we call a morphism $\mathbf{f}: \mathbf{X}=\lim _{\rightarrow} X_{n} \rightarrow \mathbf{X}^{\prime}=\lim _{\rightarrow} X_{n}^{\prime}$ of locally complete ind-varieties an embedding if all morphisms $f_{m}: X_{m} \rightarrow X_{n_{m}}^{\prime}, m \geq 1$, are embeddings.

A vector bundle $\mathbf{E}$ of rank $\mathbf{r} \in \mathbb{Z}_{>0}$ on $\mathbf{X}$ is the inverse limit $\lim _{\leftarrow} E_{m}$ of an inverse system of vector bundles $E_{m}$ or rank $\mathbf{r}$ on $X_{m}$, i.e. a system of vector bundles $E_{m}$ with fixed isomorphisms $\psi_{m}: E_{m} \cong \varphi_{m}^{*} E_{m+1}$; here and below $\varphi^{*}$ stands for inverse image of vector bundles under a morphism $\varphi$. Clearly, $\left.\mathbf{E}\right|_{X_{m}} \cong E_{m}, m \geq 1$. In particular, the structure sheaf $\mathcal{O}_{\mathbf{X}}=\lim _{\mathcal{O}_{X_{m}}}$ of an ind-variety $\mathbf{X}$ is well defined. By the Picard group PicX we understand the group of isomorphism classes of line bundles on $\mathbf{X}$. Clearly, PicX is the inverse limit $\operatorname{limPic} X_{m}$ of the inverse system $\left\{\varphi_{m}^{*}: \operatorname{Pic} X_{m+1} \rightarrow \operatorname{Pic} X_{m}\right\}_{m \geq 1}$. In the rest of the paper we automatically assume that all vector bundles considered have finite rank. If $\mathbf{E}$ is a vector bundle on $\mathbf{X}, r \mathbf{E}$ stands for the direct sum $\mathbf{E} \oplus \ldots \oplus \mathbf{E}$ of $r$ copies of $\mathbf{E}$.

A linear ind-variety is an ind-variety $\mathbf{X}=\lim _{\rightarrow} X_{m}$ such that, for each $m \geq 1$, the induced homomorphism of Picard groups $\varphi_{m}^{*}: \operatorname{Pic} X_{m+1} \rightarrow \operatorname{Pic} X_{m}$ is an epimorphism. A typical example 
of a linear ind-variety is the projective ind-space $\mathbf{P}^{\infty}$ which is the direct limit of a chain of linear embeddings

$$
\mathbb{P}^{n_{1}} \stackrel{\varphi_{1}}{\longrightarrow} \mathbb{P}^{n_{2}} \stackrel{\varphi_{2}}{\longrightarrow} \ldots{\stackrel{\varphi_{m-1}}{\hookrightarrow}}^{\varphi^{n_{m}}} \stackrel{\varphi_{m}}{\longrightarrow} \ldots
$$

for an arbitrary increasing sequence $\left\{n_{m}\right\}_{m \geq 1}$ of nonnegative integers. (It is easy to see that the definition of $\mathbf{P}^{\infty}$ does not depend, up to isomorphism of ind-varieties, on the choice of the sequence $\left\{n_{m}\right\}_{m \geq 1}$ and the embeddings $\varphi_{m}$.) By a projective ind-subspace of an ind-variety $\mathbf{X}$ we understand the image of an embedding $\psi: \mathbf{P}^{\infty} \hookrightarrow \mathbf{X}$.

Another example of a linear ind-variety is the ind-grassmannian $\mathbf{G}(\infty)$ which is the direct limit of a chain of linear embeddings

$$
G\left(k_{1}, \mathbb{C}^{n_{1}}\right) \stackrel{\varphi_{1}}{\hookrightarrow} G\left(k_{2}, \mathbb{C}^{n_{2}}\right) \stackrel{\varphi_{2}}{\hookrightarrow} \ldots \stackrel{\varphi_{m-1}}{\hookrightarrow} G\left(k_{m}, \mathbb{C}^{n_{m}}\right) \stackrel{\varphi_{m}}{\hookrightarrow} \ldots,
$$

where $G\left(k_{m}, \mathbb{C}^{n_{m}}\right)$ is the grassmannian of $k_{m}$-dimensional subspaces in an $n_{m}$-dimensional vector space and $\lim _{m \rightarrow \infty} k_{m}=\lim _{m \rightarrow \infty}\left(n_{m}-k_{m}\right)=\infty$.

Let $\mathbf{X}=\lim X_{m}$ be a linear ind-variety such that there is a finite or countable set $\Theta_{\mathbf{X}}$ and a collection $\left\{\mathbf{L}_{i}=\lim _{\leftarrow} L_{i m}\right\}_{i \in \Theta_{\mathbf{X}}}$ of line bundles on $\mathbf{X}$ such that, for any $m, L_{i m} \simeq \mathcal{O}_{X_{m}}$ for all but finitely many indices $i_{1}(m), \ldots, i_{j}(m)$, and the images of $L_{i_{1}(m) m}, \ldots, L_{i_{j}(m) m}$ in $\operatorname{Pic} X_{m}$ form a basis of $\operatorname{Pic} X_{m}$ which is assumed to be a free abelian group. It is clear that in this case PicX is isomorphic to a direct product of infinite cyclic groups with generators the images of $\mathbf{L}_{i}$. We denote by $\underset{i \in \Theta_{\mathbf{X}}}{\otimes} \mathbf{L}_{i}^{\otimes a_{i}}$ the line bundle on $\mathbf{X}$ whose restriction to $X_{m}$ equals $\otimes_{i} L_{i m}^{\otimes a_{i}}=$ $L_{i_{1}(m) m}^{\otimes a_{1}} \otimes \ldots \otimes L_{i_{j}(m) m}^{\otimes a_{j}}$. We say that $\mathbf{X}$ satisfies the property $\mathrm{L}$ if, in addition to the above condition, $H^{1}\left(X_{m}, \otimes_{i} L_{i m}^{\otimes a_{i}}\right)=0$ for any $m \geq 1$ if some $a_{i}$ is negative.

Let $\mathbf{X}$ satisfy the property $\mathrm{L}$. For a given $i \in \Theta_{\mathbf{X}}$, a smooth rational curve $C \simeq \mathbb{P}^{1}$ on $\mathbf{X}$ is a projective line of the $i$-th family on $\mathbf{X}$ (or simply, a line of the $i$-th family), if

$$
\left.\mathbf{L}_{j}\right|_{C} \cong \mathcal{O}_{\mathbb{P}^{1}}\left(\delta_{i j}\right) \quad \text { for } \quad j \in \Theta_{\mathbf{X}}
$$

By $\mathbf{B}_{i}$ we denote the set of all projective lines of the $\mathrm{i}$-th family on $\mathbf{X}$. It has a natural structure of an ind-variety: $\mathbf{B}_{i}=\lim B_{i m}$, where $B_{i m}:=\left\{C \in \mathbf{B}_{i} \mid C \subset X_{m}\right\}$ for $m \geq 1$. For any point $x \in \mathbf{X}$ the subset $\mathbf{B}_{i}(x)=\left\{C \in \mathbf{B}_{i} \mid C \ni x\right\}$ inherits an induced structure of an ind-variety.

Assume that $\mathbf{X}$ satisfies the property L. Then we say that $\mathbf{X}$ satisfies the property $\mathrm{A}$ if for any $i \in \Theta_{\mathbf{X}}$ there is an ind-variety $\Pi_{i}=\lim _{i m}$ whose points are projective ind-subspaces $\mathbf{P}^{\infty} \subset \mathbf{B}_{i}$, where $\Pi_{i m}=\left\{\mathbb{P}^{n_{m}} \subset B_{i m} \mid \mathbb{P}^{n_{m}}=\overrightarrow{\mathbf{P}}^{\infty} \cap B_{i m}\right.$ for some $\left.\mathbf{P}^{\infty} \in \Pi_{i}\right\}$, and, for any point $x \in \mathbf{X}$, the following conditions hold:

(A.i) for each $m \geq 1$ such that $x \in X_{m}$, the sheaf $L_{i m}$ defines a morphism $\psi_{i m}: X_{m} \rightarrow$ $\mathbb{P}^{r_{i m}}:=\mathbb{P}\left(H^{0}\left(X_{m}, L_{i m}\right)^{*}\right)$ which maps the family of lines $B_{i m}(x)$ isomorphically to a subfamily of lines in $\mathbb{P}^{r_{i m}}$ passing through the point $\psi_{i m}(x)$;

(A.ii) the variety $\Pi_{i m}(x):=\left\{\mathbb{P}^{n_{m}} \in \Pi_{i m} \mid \mathbb{P}^{n_{m}} \ni x\right\}$ is connected for any $m \geq 1$;

(A.iii) the projective ind-subspaces $\mathbf{P}^{\infty} \in \boldsymbol{\Pi}_{i}(x):=\lim _{\rightarrow} \prod_{i m}(x)$ fill $\mathbf{B}_{i}(x)$;

(A.iv) for any $d \in \mathbb{Z}_{\geq 1}$ there exists a $m_{0}(d) \in \mathbb{Z}_{\geq 1}$ such that, for any $d$-dimensional variety

$Y$ and any $m \geq m_{0}(d)$, any morphism $\Pi_{i m}(x) \rightarrow Y$ is a constant map.

In particular, (A.ii) and (A.iii) imply that the varieties $\Pi_{i m}, B_{i m}, B_{i m}(x)$ are connected.

Let $\mathbf{X}$ satisfy the properties $\mathrm{L}$ and $\mathrm{A}$ as above. A vector bundle $\mathbf{E}$ on $\mathbf{X}$ is called $\mathbf{B}_{i^{-} \text {uniform, }}$, if for any projective line $\mathbb{P}^{1} \in \mathbf{B}_{i}$ on $\mathbf{X}$, the restricted bundle $\left.\mathbf{E}\right|_{\mathbb{P}^{1}}$ is isomorphic to $\oplus_{j=1}^{\mathrm{rkE}} \mathcal{O}_{\mathbb{P}^{1}}\left(k_{j}\right)$ for some integers $k_{j}$ not depending on the choice of $\mathbb{P}^{1}$. If in addition all $k_{j}=0$, then $\mathbf{E}$ is called $\mathbf{B}_{i}$-linearly trivial. We call $\mathbf{E}$ uniform (respectively, linearly trivial) if it is $\mathbf{B}_{i}$-uniform (respectively, $\mathbf{B}_{i}$-linearly trivial) for any $i \in \Theta_{\mathbf{X}}$. Moreover, we say that $\mathbf{X}$ satisfies the property $\mathrm{T}$ if any linearly trivial vector bundle on $\mathbf{X}$ is trivial.

Our general version of the BVTS Theorem is the following. 
Theorem 2.1. Let $\mathbf{E}$ be a vector bundle on a linear ind-variety $\mathbf{X}$.

(i) If $\mathbf{X}$ satisfies the properties $\mathrm{L}$ and $\mathrm{A}$ for for some fixed line bundles $\left\{\mathbf{L}_{i}\right\}_{i \in \Theta_{\mathbf{X}}}$, and corresponding families $\left\{\mathbf{B}_{i}\right\}_{i \in \Theta_{\mathbf{X}}}$ of projective lines on $\mathbf{X}$, then $\mathbf{E}$ has a filtration by vector subbundles

$$
0=\mathbf{E}_{0} \subset \mathbf{E}_{1} \subset \ldots \subset \mathbf{E}_{t}=\mathbf{E}
$$

with uniform successive quotients $\mathbf{E}_{k} / \mathbf{E}_{k-1}, k=1, \ldots, t$.

(ii) If, in addition, $\mathbf{X}$ satisfies the property $T$, then the filtration (3) splits and its quotients are of the form

$$
\mathbf{E}_{k} / \mathbf{E}_{k-1} \cong \operatorname{rk}\left(\mathbf{E}_{k} / \mathbf{E}_{k-1}\right)\left(\bigotimes_{i \in \Theta_{\mathbf{X}}} \mathbf{L}_{i}^{\otimes a_{i k}}\right), \quad a_{i k} \in \mathbb{Z}, i \in \Theta_{\mathbf{X}}, 1 \leq k \leq t .
$$

In particular, $\mathbf{E}$ is isomorphic to a direct sum of line bundles.

\section{Proof OF THE MAIN THEOREM}

\subsection{Preliminaries on vector bundles.}

If $C \subset X$ is a smooth irreducible rational curve in an algebraic variety $X$ and $E$ is a vector bundle on $X$, then by a classical theorem often attributed to Grothendieck, $\left.E\right|_{C}$ is isomorphic to $\bigoplus_{i} \mathcal{O}_{C}\left(\delta_{i}\right)$ for some $\delta_{1} \geq \delta_{2} \geq \ldots \geq \delta_{\mathrm{rk} E}$. We call the ordered $\mathrm{rk} E$-tuple $\left(\delta_{1}, \ldots, \delta_{\mathrm{rk} E}\right)$ the splitting type of $\left.E\right|_{C}$ and denote it $\operatorname{Split}\left(\left.E\right|_{C}\right)$. We order splitting types lexicographically, i.e. $\left(\delta_{1}, \ldots, \delta_{\mathrm{rk} E}\right)>\left(\delta_{1}^{\prime}, \ldots, \delta_{\mathrm{rk} E}^{\prime}\right)$ if $\delta_{1}=\delta_{1}^{\prime}, \ldots, \delta_{k-1}=\delta_{k-1}^{\prime}, \delta_{k}>\delta_{k}^{\prime}$ for some $k, 1 \leq k \leq \mathrm{rkE}$.

Let $\mathbf{X}$ be a locally complete linear ind-variety satisfying the properties $\mathrm{L}$ and $\mathrm{A}$, and let $x \in \mathbf{X}$ and $i \in \Theta_{\mathbf{X}}$. In the notation of $(\mathrm{A} . \mathrm{i})$, let $\mathbb{P}^{r_{i m}}=\mathbb{P}\left(H^{0}\left(X_{m}, L_{i m}\right)^{*}\right)$ and $y=\psi_{i m}(x)=\mathbb{C} u, 0 \neq$ $u \in H^{0}\left(X_{m}, L_{i m}\right)^{*}$, so that $B_{i m}(x) \subset \mathbb{P}_{y}^{r_{i m}-1}:=\mathbb{P}\left(H^{0}\left(X_{m}, L_{i m}\right)^{*} / \mathbb{C} u\right)$. Fix a projective subspace $\mathbb{P}^{n_{m}} \subset B_{i m}(x)$, where $\mathbb{P}^{n_{m}} \in \Pi_{i m}(x)$. Then $\left.\mathcal{O}_{\mathbb{P}\left(H^{0}\left(X_{m}, L_{i m}\right)^{*} / \mathbb{C} u\right)}(1)\right|_{\mathbb{P}_{m}{ }_{m}} \simeq \mathcal{O}_{\mathbb{P}^{n} m}(N(i))$ for some $N(i)>0$. Consider the locally closed subvariety $Y_{m}:=\left\{(z, l) \in \mathbb{P}^{r_{i m}} \times \mathbb{P}^{n_{m}} \mid z \in l \backslash\{y\}\right\}$ of $\mathbb{P}^{r_{i m}} \times \mathbb{P}^{n_{m}}$, and let $Y_{m} \hookrightarrow \mathbb{P}^{r_{i m}}$ be the embedding induced by the projection $\mathbb{P}^{r_{i m}} \times \mathbb{P}^{n_{m}} \rightarrow \mathbb{P}^{r_{i m}}$. Then $Y_{m}$ is isomorphic to the total space of the line bundle $\mathcal{O}_{\mathbb{P} n_{m}}(N(i))$ (see for instance $[\mathrm{F}$, Appendix B]) and

$$
\left.\mathcal{O}_{\mathbb{P}^{r_{i m}}}(1)\right|_{Y_{m}} \simeq \pi_{m}^{*} \mathcal{O}_{\mathbb{P}^{n_{m}}}(N(i)),
$$

where $\pi_{m}: Y_{m} \rightarrow \mathbb{P}^{n_{m}}$ is the natural projection. Moreover, by construction we have a commutative diagram of morphisms

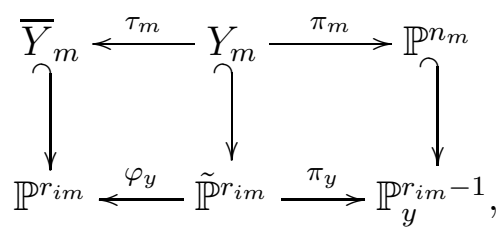

where $\tau_{m}: Y_{m} \hookrightarrow \bar{Y}_{m}:=Y_{m} \cup\{y\}$ is the inclusion, $\varphi_{y}: \tilde{\mathbb{P}}^{r_{i m}} \rightarrow \mathbb{P}^{r_{i m}}$ is the blow-up of $\mathbb{P}^{r_{i m}}$ with centre at $y$, and $\pi_{y}$ is the natural projection which is a $\mathbb{P}^{1}$-bundle. In addition, we have an open embedding

$$
\iota_{m}: Y_{m} \hookrightarrow \tilde{Y}:=\tilde{\mathbb{P}}^{r_{i m}} \times_{\mathbb{P}_{y}^{r_{i m}-1}} \mathbb{P}^{n_{m}}
$$

and projections $\bar{Y}_{m} \stackrel{\tilde{\tau}_{m}}{\leftarrow} \widetilde{Y}_{m} \stackrel{\tilde{\tau}_{m}}{\rightarrow} \mathbb{P}^{n_{m}}$ such that

$$
\tau_{m}=\tilde{\tau}_{m} \circ \iota_{m}, \quad \pi_{m}=\tilde{\pi}_{m} \circ \iota_{m} .
$$


By $($ A.i $) \psi_{i m}: \psi_{i m}^{-1}\left(\bar{Y}_{m}\right) \rightarrow \bar{Y}_{m}$ is an isomorphism. Hence we may consider $\left.\mathbf{E}\right|_{\psi_{i m}^{-1}\left(\bar{Y}_{m}\right)}$ as a vector bundle on $\bar{Y}_{m}$ and denote it by $\left.\mathbf{E}\right|_{\bar{Y}_{m}}$. We also set $\left.\mathbf{E}\right|_{Y_{m}}:=\tau_{m}^{*}\left(\left.\mathbf{E}\right|_{\bar{Y}_{m}}\right)$.

For an arbitrary projective line $\mathbb{P}^{1} \subset \mathbb{P}_{m}^{n_{m}}$, we consider the surface $S=S\left(x, \mathbb{P}^{1}\right):=\pi_{y}^{-1}\left(\mathbb{P}^{1}\right)$ with natural projections $\pi_{S}:=\left.\pi_{y}\right|_{S}: S \rightarrow \mathbb{P}^{1}$ and $\sigma_{S}:=\left.\varphi_{y}\right|_{S}: S \rightarrow \mathbf{X}$. It follows from (44) that $S$ is a surface of type $F_{N(i)}$.

Let $\mathbf{E}$ be a vector bundle of rank $\mathbf{r}$ on $\mathbf{X}$. For any $i \in \Theta_{\mathbf{X}}$ and $x \in \mathbf{X}$ we set $C(i):=$ $c_{1}\left(\left.\mathbf{E}\right|_{l}\right) \in \mathbb{Z}$, where $c_{1}$ stands for first Chern class and $l \in \mathbf{B}_{i}(x)$. Since $\mathbf{B}_{i}(x)$ is connected, $C(i)$ is well defined. Furthermore, we have $\delta_{1}\left(\left.\mathbf{E}\right|_{l}\right) \geq C(i) / \mathbf{r} \geq \delta_{B}\left(\left.\mathbf{E}\right|_{l}\right)$. Hence there are well-defined integers

$$
\delta_{1}^{\min }:=\min _{l \in \mathbf{B}_{i}(x)} \delta_{1}\left(\left.\mathbf{E}\right|_{l}\right), \quad \delta_{\mathrm{rk} E}^{\max }:=\max _{l \in \mathbf{B}_{i}(x)} \delta_{\mathrm{rk} E}\left(\left.\mathbf{E}\right|_{l}\right),
$$

and there exist lines $l_{\min }, l_{\max } \in \mathbf{B}_{i}(x)$ such that $\delta_{1}\left(\left.\mathbf{E}\right|_{l_{\min }}\right)=\delta_{1}^{\min }, \delta_{\mathrm{rk} E}\left(\left.\mathbf{E}\right|_{l_{\max }}\right)=\delta_{\mathrm{rkE}}^{\max }$. The inequality $C(i) \geq \delta_{1}^{\min }+(\mathbf{r}-1) \delta_{\mathrm{rkE}}\left(\left.\mathbf{E}\right|_{l_{\min }}\right)$ implies

$$
\delta_{1}^{\min }-\delta_{\mathrm{rk} E}\left(\left.\mathbf{E}\right|_{l_{\min }}\right) \leq \delta_{1}^{\min }-C(i) /(\mathbf{r}-1), \quad \delta_{1}\left(\left.\mathbf{E}\right|_{l_{\max }}\right)-\delta_{\mathrm{rk} E}^{\max } \geq C(i) /(\mathbf{r}-1)-\delta_{\mathrm{rk} E}^{\max } .
$$

Fix $\mathbf{P}^{\infty} \in \mathbf{B}_{i}(x)$ and $l_{\text {min }} \in \mathbf{P}^{\infty}$. For an arbitrary point $l_{0} \in \mathbf{P}^{\infty} \backslash\left\{l_{\min }\right\}$ consider the line $\mathbb{P}^{1}=\operatorname{Span}\left(l_{0}, l_{\min }\right)$ in $\mathbf{P}^{\infty}$ and the corresponding surface $S=S\left(x, \mathbb{P}^{1}\right)$ together with the vector bundle $E_{S}:=\sigma_{S}^{*} \mathbf{E}$ on $S$. For a general point $l \in \mathbb{P}^{1}(l$ is a line on $\mathbf{X})$, the first inequality in (7) implies

$$
\delta_{\text {gen }}:=\delta_{1}\left(\left.\mathbf{E}\right|_{l}\right)-\delta_{\mathrm{rk} E}\left(\left.\mathbf{E}\right|_{l}\right) \leq \delta_{1}^{\min }-C(i) /(\mathbf{r}-1) .
$$

The following lemma is a straightforward consequence of a result of Tyurin.

Lemma 3.1. There exist polynomials $P_{A}, P_{B} \in \mathbb{Q}\left[x_{1}, \ldots, x_{6}\right]$ such that for any $l_{0} \in \mathbf{P}^{\infty} \backslash\left\{l_{\min }\right\}$

$$
\begin{gathered}
\delta_{1}\left(\left.\mathbf{E}\right|_{l_{0}}\right) \leq P_{A}\left(\mathbf{r}, \delta_{1}^{\min }, C(i), N(i), c_{1}^{2}\left(E_{S}\right), c_{2}\left(E_{S}\right)\right)=: P_{A}(\mathbf{E}, i), \\
\delta_{\mathrm{rk} E}\left(\left.\mathbf{E}\right|_{l_{0}}\right) \geq P_{B}\left(\mathbf{r}, \delta_{\mathrm{rk} E}^{\max }, C(i), N(i), c_{1}^{2}\left(E_{S}\right), c_{2}\left(E_{S}\right)\right)=: P_{B}(\mathbf{E}, i),
\end{gathered}
$$

where $c_{1}^{2}\left(E_{S}\right)$ and $c_{2}\left(E_{S}\right)$ are considered as integers.

Proof. By construction, $S$ is a surface of type $F_{N(i)}$. Hence, repeating for the vector bundle $E_{S}$ the proof of Lemma 5 from $[\mathrm{T}$, Ch. $2, \S 1]$ we obtain that there exists a polynomial $f \in$ $\mathbb{Q}\left[x_{1}, \ldots, x_{6}\right]$ such that $\delta_{1}\left(\left.\mathbf{E}\right|_{l_{0}}\right) \leq f\left(\mathbf{r}, \delta_{1}^{\min }, \delta_{\text {gen }}, N(i), c_{1}^{2}\left(E_{S}\right), c_{2}\left(E_{S}\right)\right)$. Thus, in view of (8) $)$, there exists a polynomial $P_{A} \in \mathbb{Q}\left[x_{1}, \ldots, x_{6}\right]$ satisfying (9). The proof of (10) is similar.

The next proposition employs in a crucial way results of E. Sato. Fix $i \in \Theta_{\mathbf{X}}, x \in \mathbf{X}$ and $\mathbb{P}^{n_{m}} \in \Pi_{i m}(x)$ for a large enough $m$. In view of (9) there exists a maximal (with respect to lexicographic order) splitting type $S_{i}\left(\mathbf{E}, \mathbb{P}^{n_{m}}\right):=\max _{l \in \mathbb{P}_{m}} \operatorname{Split}\left(\left.\mathbf{E}\right|_{l}\right)$.

Proposition 3.2. The maximal splitting type $S_{i}\left(\mathbf{E}, \mathbb{P}^{n_{m}}\right)$ depends only on the pair $(\mathbf{E}, i)$, i.e. $S_{i}\left(\mathbf{E}, \mathbb{P}^{n_{m}}\right)$ does not depend on $x$ and on $\mathbb{P}^{n_{m}} \in \Pi_{i m}(x)$.

Proof. Set

$$
M_{i}\left(\mathbb{P}^{n_{m}}\right):=\left\{l \in \mathbb{P}^{n_{m}} \mid \operatorname{Split}\left(\left.\mathbf{E}\right|_{l}\right)=S_{i}\left(\mathbf{E}, \mathbb{P}^{n_{m}}\right)\right\}
$$

The semicontinuity of $\operatorname{Split}\left(\left.\mathbf{E}\right|_{l}\right)$ implies that $M_{i}\left(\mathbb{P}^{n_{m}}\right)$ is a closed subvariety of $\mathbb{P}^{n_{m}}$. Moreover, Lemma 3.1 together with [T, Ch. 2, §2, Lemmas 3 and 4] yields the inequality

$$
\operatorname{codim}_{\mathbb{P}^{n} m} M_{i}\left(\mathbb{P}^{n_{m}}\right) \leq \mathbf{r}(\mathbf{r}-1)\left(P_{A}(\mathbf{E}, i)-P_{B}(\mathbf{E}, i)\right) .
$$

Consider the upper row of the diagram (5). Since the right-hand side of (11) is constant with respect to $m$, for large enough $m$ we have

$$
\operatorname{codim}_{\mathbb{P}^{n_{m}}} M_{i}\left(\mathbb{P}^{n_{m}}\right)<\min \left(n_{m}-\mathbf{r},\left(n_{m}-2 \mathbf{r}^{2}\right) / 2\right) .
$$

Also, clearly for large enough $m$

$$
\operatorname{codim}_{\bar{Y}_{m}}\{x\}=\operatorname{codim}_{\bar{Y}_{m}}\left(\bar{Y}_{m} \backslash Y_{m}\right)>\mathbf{r} .
$$


The inequality (13) shows that

$$
c_{k}\left(\left.\mathbf{E}\right|_{Y_{m}}\right)=\tau_{m}^{*} c_{k}\left(\left.\mathbf{E}\right|_{\bar{Y}_{m}}\right), \quad 0 \leq k \leq \mathbf{r},
$$

where $c_{k}(\cdot)$ stands for $k$-th Chern class. Moreover, since the Chow group $A^{k}(\cdot)$ of codimension $k$ pulls back isomorphically to the total space of any vector bundle, we have

$$
c_{k}\left(\left.\mathbf{E}\right|_{Y_{m}}\right)=\pi_{m}^{*}\left(c_{k} H^{k}\right), \quad 0 \leq k \leq \mathbf{r},
$$

where $H$ is the class of a hyperplane divisor on $\mathbb{P}^{n_{m}}$ and $c_{1}, \ldots, c_{\mathbf{r}}$ are integers. It is essential to note that the obvious compatibility of the morphisms $\pi_{m}$ for varying $m$ and the functoriality of Chern classes imply that these integers do not depend on $x$ and on $\mathbb{P}^{n_{m}} \in \Pi_{i m}(x)$. Note also that (41), (6) and the equalities (14) and (15) imply

$\iota_{m}^{*} c_{k}\left(\tilde{\tau}_{m}^{*}\left(\left.\mathbf{E} \otimes \mathbf{L}_{i}^{-a}\right|_{\bar{Y}_{m}}\right)\right)=\tau_{m}^{*} c_{k}\left(\left.\mathbf{E} \otimes \mathbf{L}_{i}^{-a}\right|_{\bar{Y}_{m}}\right)=c_{k}\left(\left.\mathbf{E}\right|_{Y_{m}} \otimes \pi_{m}^{*} \mathcal{O}_{\mathbb{P}^{n} m}(-N(i) a H)\right), \quad 0 \leq k \leq \mathbf{r}, a \in \mathbb{Z}$.

Next, consider the polynomial

$$
h(t)=\sum_{k=0}^{\mathbf{r}} c_{k}(-t)^{\mathbf{r}-k} \in \mathbb{Z}[t]
$$

where the coefficients $c_{k}$ are the integers introduced above. Following closely an idea of Sato, we will now argue that the roots of $h(t)$ constitute a constant multiple of the maximal splitting type $S_{i}\left(\mathbf{E}, \mathbb{P}^{n_{m}}\right)$. More precisely, let $a_{1}>\ldots>a_{\alpha}, \alpha \leq \mathbf{r}$, be the distinct elements of $S_{i}\left(\mathbf{E}, \mathbb{P}^{n_{m}}\right)$ of respective multiplicities $r_{1}, \ldots, r_{\alpha}$ in $S_{i}\left(\mathbf{E}, \mathbb{P}^{n_{m}}\right)$. Then we claim that the roots of $h(t)$ are $N(i) a_{1}, \ldots, N(i) a_{\alpha}$ of respective multiplicities $r_{1}, \ldots, r_{\alpha}$.

The argument in [S1, pp. 138-139] shows that in order to prove this claim of $h(t)$ it suffices to establish the vanishing of $c_{k}\left(\left.\mathbf{E}\right|_{Y_{m}} \otimes \pi_{m}^{*} \mathcal{O}_{\mathbb{P}^{n}}\left(-N(i) a_{j} H\right)\right)$ for $\mathbf{r}-r_{j}+1 \leq k \leq \mathbf{r}, 1 \leq j \leq \alpha$. By (16) it is enough to prove the vanishing of $c_{k}\left(\tilde{\tau}_{m}^{*}\left(\left.\mathbf{E} \otimes \mathbf{L}_{i}^{-a_{j}}\right|_{\bar{Y}_{m}}\right)\right)$. However, the proof of this fact is practically the same as in [S1]. Namely, one defines inductively vector bundles $F_{1}:=\left.\tilde{\varphi}_{m}^{*} \mathbf{E}\right|_{\tilde{\pi}_{m}^{-1}\left(M_{i}\left(\mathbb{P}^{n} m\right)\right)}, F_{2}, \ldots, F_{\alpha}$ such that $\mathrm{rk} F_{j}=\sum_{p=j}^{\alpha} r_{p}$ on $\tilde{\pi}_{m}^{-1}\left(M_{i}\left(\mathbb{P}^{n_{m}}\right)\right)$ which fit into the exact triples

$$
0 \rightarrow r_{j} \mathcal{O}_{\tilde{\pi}_{m}^{-1}\left(M_{i}\left(\mathbb{P}^{n m}\right)\right)} \rightarrow F_{j} \otimes\left(\left.\mathbf{L}_{i}^{a_{j-1}-a_{j}}\right|_{\tilde{\pi}_{m}^{-1}\left(M_{i}\left(\mathbb{P}^{n}\right)\right)}\right) \rightarrow F_{j+1} \rightarrow 0, \quad 1 \leq j \leq \alpha-1,
$$

where $a_{0}:=0$. Using (12) and applying the argument from [S1, p. 139] to the triples (18), we obtain $c_{k}\left(\tilde{\tau}_{m}^{*}\left(\left.\mathbf{E} \otimes \mathbf{L}_{i}^{-a_{j}}\right|_{\bar{Y}_{m}}\right)\right)=0$ as desired.

Since $h(t)$ is independent of $x$ and $\mathbb{P}^{n_{m}} \in \Pi_{i m}(x)$, the same applies to $S_{i}\left(\mathbf{E}, \mathbb{P}^{n_{m}}\right)$, i.e. the proposition is proved.

\subsection{Proof of Theorem 2.1.}

Proof. According to (12), the dimension of $M_{i}\left(\mathbb{P}^{n_{m}}\right)$ is greater than half of the dimension of $\mathbb{P}^{n_{m}}$, hence the varieties $M_{i}\left(\mathbb{P}^{n_{m}}\right)$ are connected for large enough $m$. Consider the variety $\Gamma_{i m}(x):=$ $\left\{\left(l, \mathbb{P}^{n_{m}}\right) \in B_{i m}(x) \times \Pi_{i m}(x) \mid l \in M_{i}\left(\mathbb{P}^{n_{m}}\right)\right\}$ with projections $B_{i m}(x) \stackrel{p_{1}}{\leftarrow} \Gamma_{i m}(x) \stackrel{p_{2}}{\rightarrow} \prod_{i m}(x)$. Since $M_{i}\left(\mathbb{P}^{n_{m}}\right)=p_{2}^{-1}\left(\mathbb{P}^{n_{m}}\right)$ is connected for any $\mathbb{P}^{n_{m}} \in \Pi_{i m}(x)$ and $\Pi_{i m}(x)$ is connected by (A.ii), it follows that $\Gamma_{i m}(x)$ is connected. By definition, $\Gamma_{i m}(x)$ is described as

$$
\Gamma_{i m}(x)=\underset{\mathbb{P}^{n} m \in \Pi_{i m}(x)}{\sqcup} M_{i}\left(\mathbb{P}^{n_{m}}\right), \quad m \geq 1 .
$$

Similarly, consider the varieties $\Gamma_{i m}:=\left\{\left(x, l, \mathbb{P}^{n_{m}}\right) \in X_{m} \times B_{i m} \times \Pi_{i m} \mid\left(l, \mathbb{P}^{n_{m}}\right) \in \Gamma_{i m}(x)\right\}$. By construction,

$$
\Gamma_{i m}=\underset{x \in X_{m}}{\sqcup} \Gamma_{i m}(x),
$$

so that each $\Gamma_{i m}$ is connected. Moreover, there is a well-defined ind-variety $\Gamma_{i}:=\underset{\rightarrow}{\lim } \Gamma_{i m}$. 
Let $\left(x, l, \mathbb{P}^{n_{m}}\right) \in \boldsymbol{\Gamma}_{i}$. If $\delta_{1}^{\max }$ is the maximal entry of $\operatorname{Split}\left(\left.\mathbf{E}\right|_{l}\right)=S_{i}\left(\mathbf{E}, \mathbb{P}^{n_{m}}\right)$, there is a well-defined subbundle $\mathbf{E}_{1}(l)$ of $\left.\mathbf{E}\right|_{l}$ :

$$
\mathbf{E}_{1}(l):=\operatorname{im}\left(\left.H^{0}\left(l,\left.\mathbf{E}\right|_{l}\left(-\delta_{1}^{\max }\right)\right) \otimes \mathcal{O}_{l} \stackrel{e v}{\rightarrow} \mathbf{E}\right|_{l}\left(-\delta_{1}^{\max }\right)\right) \otimes \mathcal{O}_{l}\left(\delta_{1}^{\max }\right) .
$$

Set $\mathbf{r}_{1}:=\mathrm{rkE}_{1}(l)$ and consider the relative grassmannian $\rho_{1}: \mathbf{G}\left(\mathbf{r}_{1}, \mathbf{E}\right) \rightarrow \mathbf{X}$. According to Proposition [3.2, $\delta_{1}^{\max }$ and $\mathbf{r}_{1}$ do not depend on the point $\left(x, l, \mathbb{P}^{n_{m}}\right) \in \boldsymbol{\Gamma}_{i}$. Thus there is a morphism of ind-varieties

$$
\mathbf{f}_{i 1}: \boldsymbol{\Gamma}_{i} \rightarrow \mathbf{G}\left(\mathbf{r}_{1}, \mathbf{E}\right),\left.\quad(x, l) \mapsto \mathbf{E}_{1}(l)\right|_{x}, \quad x \in \mathbf{X} .
$$

Since $\mathbf{f}_{i 1}\left(\Gamma_{i m}(x)\right) \subset \rho_{1}^{-1}(x)=G\left(\mathbf{r}_{1},\left.\mathbf{E}\right|_{x}\right)$, by (19) we have

$$
\mathbf{f}_{i 1}\left(M_{i}\left(\mathbb{P}^{n_{m}}\right)\right) \subset G\left(\mathbf{r}_{1},\left.\mathbf{E}\right|_{x}\right), \quad \mathbb{P}^{n_{m}} \in \Pi_{i m}(x) .
$$

According to (11) $\operatorname{codim}_{\mathbb{P}^{n_{m}}} M_{i}\left(\mathbb{P}^{n_{m}}\right)$ is bounded as $m \rightarrow \infty$. This means that, for large enough $m$, the morphism $\mathbf{f}_{i 1}: M_{i}\left(\mathbb{P}^{n_{m}}\right) \rightarrow G\left(\mathbf{r}_{1},\left.\mathbf{E}\right|_{x}\right)$ satisfies the conditions of [S1, Prop. 3.2], in which we set $n=n_{m}, X=M_{i}\left(\mathbb{P}^{n_{m}}\right), Y=G\left(\mathbf{r}_{1},\left.\mathbf{E}\right|_{x}\right)$ and $f=\mathbf{f}_{i 1}$. By this proposition, $\left.\mathbf{f}_{i 1}\right|_{M_{i}\left(\mathbb{P}^{n m}\right)}$ is a constant map, hence it induces a morphism

$$
\varphi_{i 1}(x): \Pi_{i m}(x) \rightarrow G\left(\mathbf{r}_{1},\left.\mathbf{E}\right|_{x}\right), \quad \mathbb{P}^{n_{m}} \mapsto \mathbf{f}_{i 1}\left(M_{i}\left(\mathbb{P}^{n_{m}}\right)\right), \quad x \in \mathbf{X} .
$$

Now (A.iv) implies that there exists a positive integer $m_{1}$ such that the morphism $\varphi_{i 1}(x)$ is a constant map for any $m \geq m_{1}$. We thus obtain that (21) induces a constant morphism

$$
\boldsymbol{\varphi}_{i 1}(x): \boldsymbol{\Pi}_{i}(x) \rightarrow G\left(\mathbf{r}_{1},\left.\mathbf{E}\right|_{x}\right) .
$$

Consider the ind-variety $\boldsymbol{\Sigma}_{i}=\lim _{\rightarrow} \Sigma_{i m}$, where $\Sigma_{i m}:=\left\{\left(x, \mathbb{P}^{n_{m}}\right) \in X_{m} \times \Pi_{i m} \mid \mathbb{P}^{n_{m}} \in \Pi_{i m}(x)\right\}$, and let $\mathbf{p}_{i}: \boldsymbol{\Sigma}_{i} \rightarrow \mathbf{X}$ be the natural projection with fibre $\boldsymbol{\Pi}_{i}(x), x \in \mathbf{X}$. The above constant morphisms $\boldsymbol{\varphi}_{i 1}(x)$ extend to a morphism $\boldsymbol{\varphi}_{i 1}: \boldsymbol{\Sigma}_{i} \rightarrow \mathbf{G}\left(\mathbf{r}_{1}, \mathbf{E}\right)$ which is constant on the fibres of $\mathbf{p}_{i}$. In addition, the morphism $\left.\mathbf{f}_{i 1}\right|_{\mathbf{M}_{i}(x)}$ is a constant map. We thus obtain a well-defined morphism

$$
\mathbf{\Phi}_{i 1}: \mathbf{X} \rightarrow \mathbf{G}\left(\mathbf{r}_{1}, \mathbf{E}\right), \quad x \mapsto \mathbf{f}_{i 1}\left(\boldsymbol{\Gamma}_{i}(x)\right) .
$$

Let $\mathcal{S}$ be the tautological bundle of $\operatorname{rank} \mathbf{r}_{1}$ on $\mathbf{G}\left(\mathbf{r}_{1}, \mathbf{E}\right)$. Set $\mathbf{E}_{1 i}:=\boldsymbol{\Phi}_{i 1}^{*} \mathcal{S}$. It follows now from (20), (21) and (22) that $\mathbf{E}_{1 i}$ is a subbundle of $\mathbf{E}$ such that

$$
\left.\mathbf{E}_{1 i}\right|_{l}=\mathbf{E}_{1}(l) \simeq \mathbf{r}_{1} \mathcal{O}_{l}\left(\delta_{1}^{\max }\right), \quad l \in \mathbf{M}_{i} .
$$

Using the semicontinuity of $\operatorname{dim} H^{0}\left(l,\left.\mathbf{E}_{1 i}\left(-\delta_{1}^{\max }\right)\right|_{l}\right)$, one checks immediately that the last inequality is true for any $l \in \mathbf{B}_{i}$.

Applying the above argument to the quotient $\mathbf{E}^{\prime}=\mathbf{E} / \mathbf{E}_{1 i}$ etc., we obtain a filtration of the bundle $\mathbf{E}$

$$
0 \subset \mathbf{E}_{1 i} \subset \mathbf{E}_{2 i} \subset \ldots \subset \mathbf{E}_{\alpha i}=\subset \mathbf{E}_{1 i}
$$

with $\mathbf{B}_{i}$-uniform successive quotients $\mathbf{F}_{k i}=\mathbf{E}_{k i} / \mathbf{E}_{k-1, i}$.

Fix now $j \in \Theta_{\mathbf{X}}, i \neq j$. By applying the same procedure to all bundles $\mathbf{F}_{k i}$, we obtain a bundle filtration of $\mathbf{E}$ whose quotients are $\mathbf{B}_{i}$-uniform and $\mathbf{B}_{j}$-uniform. After finitely many iterations we finally obtain a filtration

$$
0=\mathbf{E}_{0} \subset \mathbf{E}_{1} \subset \ldots \subset \mathbf{E}_{s}=\mathbf{E}
$$

of $\mathbf{E}$ with uniform successive quotients. This yields (i).

Note that any uniform vector bundle on $\mathbf{X}$ becomes linearly trivial after twisting by an appropriate line bundle. This means that each successive quotient $\mathbf{E}_{k} / \mathbf{E}_{k-1}$ is isomorphic to $\mathbf{M}_{k} \otimes \mathbf{F}_{k}$ where $\mathbf{M}_{k}$ is a line bundle and $\mathbf{F}_{k}$ is linearly trivial. In addition, assume that the property $\mathrm{T}$ is satisfied. Then the bundles $\mathbf{F}_{k}$ are trivial, i.e.

$$
\mathbf{E}_{k} / \mathbf{E}_{k-1} \simeq \operatorname{rk}\left(\mathbf{E}_{k} / \mathbf{E}_{k-1}\right) \mathbf{M}_{k}, \quad 1 \leq k \leq s
$$

Furthermore, for $p<k$

$$
\operatorname{Ext}^{1}\left(\mathbf{M}_{k}, \mathbf{M}_{p}\right)=H^{1}\left(\mathbf{X}, \mathbf{M}_{k}^{*} \otimes \mathbf{M}_{p}\right)
$$


and, according to a well known fact [H3, Theorem 4.5] (see also [DPW, Proposition 10.3]),

$$
H^{1}\left(\mathbf{X}, \mathbf{M}_{k}^{*} \otimes \mathbf{M}_{p}\right)=\lim _{\leftarrow} H^{1}\left(X_{m},\left.\left(\mathbf{M}_{k}^{*} \otimes \mathbf{M}_{p}\right)\right|_{X_{m}}\right) .
$$

However, the above construction shows that

$$
\left.\left(\mathbf{M}_{k}^{*} \otimes \mathbf{M}_{p}\right)\right|_{X_{m}} \simeq \otimes_{i} L_{i m_{i}}^{\otimes a_{i}}
$$

with some $a_{i}$ negative. Therefore the vanishing part of property L yields

$$
H^{1}\left(X_{m},\left.\left(\mathbf{M}_{k}^{*} \otimes \mathbf{M}_{p}\right)\right|_{X_{m}}\right)=0
$$

for $m \geq 1$, and hence $\operatorname{Ext}^{1}\left(\mathbf{M}_{k}, \mathbf{M}_{p}\right)=0$ for $1 \leq p \leq k \leq s$. This is sufficient to conclude that the filtration (23) splits, i.e. (ii) follows.

The rest of the paper (with exception of the appendix) is devoted to examples of linear ind-varieties satisfying the properties L, A and T.

\section{Linear ind-GRASSmannians SATisfying the properties L, A, T}

\subsection{Finite-dimensional orthogonal and symplectic grassmannians.}

Let $V$ be a finite-dimensional vector space. In what follows we will consider, both symmetric and symplectic, quadratic forms $\Phi$ on $V$. Under the assumption that $\Phi$ is fixed, for any subspace $W \subset V$ we set $W^{\perp}:=\{v \in V \mid \Phi(v, w)=0$ for any $w \in W\}$. Recall that $W$ is isomorphic (or $\Phi$-isomorphic) if $W \subset W^{\perp}$.

Let $\Phi \in S^{2} V^{*}$ be a non-degenerate symmetric form on $V$. For $\operatorname{dim} V \geq 3$ and $1 \leq k \leq\left[\frac{\operatorname{dim} V}{2}\right]$, the orthogonal grassmannian $G O(k, V)$ is defined as the subvariety of $G(k, V)$ consisting of all $\Phi$-isotropic $k$-dimensional subspaces of $V$. Unless $\operatorname{dim} V=2 n, k=n, G O(k, V)$ is a smooth irreducible variety. For $\operatorname{dim} V=2 n, k=n, G O(k, V)$ is smooth and has two irreducible components, both of which are isomorphic to $G O(n-1, \tilde{V})$ for $\operatorname{dim} \tilde{V}=2 n-1$.

If $\Phi \in \wedge^{2} V^{*}$ is a non-degenerate symplectic form on $V, \operatorname{dim} V=2 n$, we recall that the symplectic grassmannian $G S(k, V)$ is a smooth irreducible subvariety of $G(k, V)$ consisting of all $\Phi$-isotropic $k$-dimensional subspaces of $V$.

\subsection{Definition of linear ind-grassmannians.}

We start by recalling the definition of standard extension of grassmannians [PT3].

By a standard extension of grassmannians we understand an embedding of grassmannians $f: G(k, V) \rightarrow G\left(k, V^{\prime}\right)$ for $\operatorname{dim} V \geq \operatorname{dim} V, k^{\prime} \geq k$, given by the formula

$$
f: V_{k} \mapsto V_{k} \oplus W
$$

for some fixed isomorphism $V^{\prime} \simeq V \oplus \hat{W}$ and a fixed subspace $W \subset \hat{W}$ of dimension $k^{\prime}-k$. Respectively, by a standard extension of orthogonal respectively, of symplectic grassmannians we understand an embedding of isotropic grassmannians $f: G O(k, V) \rightarrow G O\left(k, V^{\prime}\right)$ (respectively, $\left.f: G S(k, V) \rightarrow G S\left(k, V^{\prime}\right)\right)$ given by the formula (24) for some fixed orthogonal (respectively, symplectic) isomorphism $V^{\prime} \simeq V \oplus \hat{W}$ and a fixed isotropic subspace $W \subset \hat{W}$ of dimension $k^{\prime}-k$, cf. [PT3, Definitions 3.2. and 3.5]. Note that standard extensions are linear morphisms.

Next we recall the definition of a standard ind-grassmannian [PT3].

Definition 4.1. Fix an infinite chain of vector spaces

$$
V_{n_{1}} \subset V_{n_{2}} \subset \ldots \subset V_{n_{m}} \subset V_{n_{m+1}} \subset \ldots
$$

of dimensions $n_{m}, n_{m}<n_{m+1}$. 
a) For an integer $k, 1 \leq k<n_{1}$, set $\mathbf{G}(k):=\lim _{\rightarrow} G\left(k, V_{n_{m}}\right)$ where

$$
G\left(k, V_{n_{1}}\right) \hookrightarrow G\left(k, V_{n_{2}}\right) \hookrightarrow \ldots \hookrightarrow G\left(k, V_{n_{m}}\right) \hookrightarrow G\left(k, V_{n_{m+1}}\right) \hookrightarrow \ldots
$$

is the chain of canonical inclusions of grassmannians induced by (25).

b) For a sequence of integers $1 \leq k_{1}<k_{2}<\ldots$ such that $k_{m}<n_{m}, \lim _{m \rightarrow \infty}\left(n_{m}-k_{m}\right)=\infty$, set $\mathbf{G}(\infty):=\lim _{\rightarrow} G\left(k_{m}, V_{n_{m}}\right)$ where

$$
G\left(k_{1}, V_{n_{1}}\right) \hookrightarrow G\left(k_{2}, V_{n_{2}}\right) \hookrightarrow \ldots \hookrightarrow G\left(k_{m}, V_{n_{m}}\right) \hookrightarrow G\left(k_{m+1}, V_{n_{m+1}}\right) \hookrightarrow \ldots
$$

is an arbitrary chain of standard extensions of grassmannians.

c) Assume that $V_{n_{m}}$ are endowed with compatible non-degenerate symmetric (respectively, symplectic) forms $\Phi_{m}$. In the symplectic case $\frac{n_{m}}{2} \in \mathbb{Z}_{+}$. For an integer $k, 1 \leq k \leq\left[\frac{n_{1}}{2}\right]$, set $\operatorname{GO}(k, \infty):=\lim _{\rightarrow} G O\left(k, V_{n_{m}}\right)$ (respectively, $\mathbf{G S}(k, \infty):=\lim _{\rightarrow} G S\left(k, V_{n_{m}}\right)$ ) where

$$
G O\left(k, V_{n_{1}}\right) \hookrightarrow G O\left(k, V_{n_{2}}\right) \hookrightarrow \ldots \hookrightarrow G O\left(k, V_{n_{m}}\right) \hookrightarrow G O\left(k, V_{n_{m+1}}\right) \hookrightarrow \ldots
$$

(respectively,

$$
\left.G S\left(k, V_{n_{1}}\right) \hookrightarrow G S\left(k, V_{n_{2}}\right) \hookrightarrow \ldots \hookrightarrow G S\left(k, V_{n_{m}}\right) \hookrightarrow G S\left(k, V_{n_{m+1}}\right) \hookrightarrow \ldots\right)
$$

is the chain of inclusions of isotropic grassmannians induced by (25).

d) For a sequence of integers $1 \leq k_{1}<k_{2}<\ldots$ such that $k_{m}<\left[\frac{n_{m}}{2}\right], \lim _{m \rightarrow \infty}\left(\left[\frac{n_{m}}{2}\right]-k_{m}\right)=\infty$, set $\mathrm{GO}(\infty, \infty)=\lim _{\rightarrow} G \mathrm{O}\left(k_{m}, V_{n_{m}}\right)$ (respectively, $\mathrm{GS}(\infty, \infty):=\lim _{\rightarrow} G S\left(k_{m}, V_{n_{m}}\right)$ ) where

$$
G O\left(k_{1}, V_{n_{1}}\right) \hookrightarrow G O\left(k_{2}, V_{n_{2}}\right) \hookrightarrow \ldots \hookrightarrow G O\left(k_{m}, V_{n_{m}}\right) \hookrightarrow G O\left(k_{m+1}, V_{n_{m+1}}\right) \hookrightarrow \ldots
$$

(respectively,

$$
\left.G S\left(k_{1}, V_{n_{1}}\right) \hookrightarrow G S\left(k_{2}, V_{n_{2}}\right) \hookrightarrow \ldots \hookrightarrow G S\left(k_{m}, V_{n_{m}}\right) \hookrightarrow G S\left(k_{m+1}, V_{n_{m+1}}\right) \hookrightarrow \ldots\right)
$$

is an arbitrary chain of standard extensions of isotropic grassmannians.

e) In the symplectic case, consider a sequence of integers $1 \leq k_{1}<k_{2}<\ldots$ such that $k_{m} \leq \frac{n_{m}}{2}, \lim _{m \rightarrow \infty}\left(\frac{n_{m}}{2}-k_{m}\right)=k \in \mathbb{N}$, and set $\mathrm{GS}(\infty, k):=\lim _{\rightarrow} G S\left(k_{m}, V_{n_{m}}\right)$ for any chain of standard extensions (27). In the orthogonal case, assume first that $\operatorname{dim} V_{n_{m}}$ are even. Then set $\mathrm{GO}^{0}(\infty, k):=\lim _{\rightarrow} G O\left(k_{m}, V_{n_{m}}\right)$ for a chain (26) where $k_{m}<\frac{n_{m}}{2}, \lim _{m \rightarrow \infty}\left(\frac{n_{m}}{2}-k_{m}\right)=k \in \mathbb{N}, k \geq 2$. Finally, consider the orthogonal case under the assumption that $\operatorname{dim} V_{n_{m}}$ are odd. Then set $\mathrm{GO}^{1}(\infty, k):=\lim _{\rightarrow} G O\left(k_{m}, V_{n_{m}}\right)$ for a chain (26) where $k_{m} \leq\left[\frac{n_{m}}{2}\right], \lim _{m \rightarrow \infty}\left(\left[\frac{n_{m}}{2}\right]-k_{m}\right)=k \in \mathbb{N}$.

In particular, $\mathbf{P}^{\infty}=\mathbf{G}(1) \simeq \mathbf{G S}(1)$. Note that the above standard ind-grassmannians are well-defined, i.e. a standard ind-grassmannian does not depend, up to an isomorphism of indvarieties, on the specific chain of standard embeddings used in its definition. Furthermore, the main result of [PT3] claims that, with the exception of the isomorphism $\mathbf{P}^{\infty} \simeq \operatorname{GS}(1)$, the standard ind-grassmannians are pairwise non-isomorphic as ind-varieties.

In all cases the maximal exterior power of a tautological bundle generated by its global sections yields an ample line bundle $\mathcal{O}_{X_{m}}(1)$, where $X_{m}=G\left(k_{m}, V_{n_{m}}\right), G O\left(k_{m}, V_{n_{m}}\right), G S\left(k_{m}, V_{n_{m}}\right)$. It is well-known that $\mathcal{O}_{X_{m}}(1)$ generates $\operatorname{Pic} X_{m}$. Moreover, if $i_{m}: X_{m} \hookrightarrow X_{m+1}$ is one of the embeddings in Definition 4.1, there is an isomorphism $i_{m}^{*} \mathcal{O}_{X_{m+1}}(1) \simeq \mathcal{O}_{X_{m}}(1)$. This allows us to conclude that $\mathbf{X}=\lim _{\rightarrow} X_{m}$ is a linear ind-variety and PicX is generated by $\mathcal{O}_{\mathbf{X}}(1):=\lim _{\leftarrow} \mathcal{O}_{X_{m}}(1)$.

4.3. BVTS Theorem for $\mathrm{G}(\infty), \mathrm{GO}(\infty, \infty), \mathrm{GS}(\infty, \infty), \mathrm{GO}^{1}(\infty, 0)$ and $\mathrm{GS}(\infty, 0)$.

We first note that, if $\mathbf{X}=\mathbf{G}(k), \mathbf{G O}(k, \infty), \mathbf{G S}(k, \infty)$ there is a tautological rank- $k$ bundle $\mathbf{S}$ on $\mathbf{X}$. If $k \geq 2$, this bundle is not isomorphic to a direct sum of line bundles, hence the BVTS theorem does not hold for these ind-grassmannians. Moreover, it is known [S2] that, for $\mathbf{X}=\mathbf{G}(k), \mathbf{G O}(k, \infty), \mathbf{X}=\mathbf{G S}(k, \infty)$, any simple vector bundle of finite rank on $\mathbf{X}$, i.e. a vector bundle which does not have a non-trivial proper subbundle, is a direct summand in a tensor power of $\mathbf{S}$. 
Theorem 4.2. Any vector bundle $\mathbf{E}$ on $\mathbf{X} \simeq \mathbf{G}(\infty), \mathbf{G O}(\infty, \infty), \mathbf{G S}(\infty, \infty), \mathbf{G O}^{1}(\infty, 0)$, $\mathrm{GS}(\infty, 0)$ is isomorphic to $\underset{i}{\oplus \mathcal{O}_{\mathbf{X}}}\left(k_{i}\right)$ for some $k_{i} \in \mathbb{Z}$.

For $\mathbf{X}=\mathbf{G}(\infty)$ this is proved in [DP] (see also [PT1, Section 4]). For the remaining standard ind-grassmannians the claim of Theorem 4.2 follows from Theorem 2.1 and the following theorem.

Theorem 4.3. Let $\mathbf{X} \simeq \mathrm{GO}(\infty, \infty), \mathbf{G S}(\infty, \infty), \mathbf{G O}^{1}(\infty, 0)$ or $\mathbf{G S}(\infty, 0)$. Then $\mathbf{X}$ satisfies the properties $\mathrm{L}, \mathrm{A}$ and $\mathrm{T} 1 \mathrm{I}$

Proof. $\mathbf{X}$ satisfies the property $\mathrm{L}$ as $\mathcal{O}_{\mathbf{X}}(1)$ generates $\operatorname{Pic} \mathbf{X}$, and $H^{1}\left(X_{m}, \mathcal{O}_{X_{m}}(a)\right)$ vanishes for all $a$ and sufficiently large $m$ by Borel-Weil-Bott's Theorem. Furthermore, the property T follows from Proposition 7.4 below.

It remains to establish the property A. Part (A.i) holds here simply because $\mathcal{O}_{\mathbf{X}}(1)$ is very ample. We therefore discuss parts (A.ii)-(A.iv).

Let $\mathbf{X}=\mathbf{G O}(\infty, \infty)=\lim G O\left(k_{m}, V_{n_{m}}\right)$. For $m \geq 1$, the base $B_{m}$ of the family of projective lines on $G O\left(k_{m}, V_{n_{m}}\right)$ coincides with the variety of isotropic flags of type $\left(k_{m}-1, k_{m}+1\right)$ in $V_{n_{m}}$ :

$$
B_{m}=\left\{\left(V_{k_{m}-1}, V_{k_{m}+1}\right) \in G O\left(k_{m}-1, V_{n_{m}}\right) \times G O\left(k_{m}+1, V_{n_{m}}\right) \mid V_{k_{m}-1} \subset V_{k_{m}+1}\right\}
$$

[PT3, Lemma 2.2(i)]. Furthermore, set

$$
\Pi_{m}:=\left\{\left(V_{k_{m}}, V_{k_{m}+1}\right) \in G O\left(k_{m}, V_{n_{m}}\right) \times G O\left(k_{m}+1, V_{n_{m}}\right) \mid V_{k_{m}} \subset V_{k_{m}+1}\right\} .
$$

A point $y=\left(V_{k_{m}}, V_{k_{m}+1}\right) \in \Pi_{m}$ corresponds to the projective subspace $G\left(k_{m}-1, V_{n_{m}}\right) \times$ $\left\{V_{k_{m}+1}\right\} \subset B_{m}$.

It is easy to see that $\Pi:=\lim \Pi_{m}$ is a well-defined ind-variety and that a point of $\Pi$ represents a projective ind-subspace of $\overrightarrow{\mathbf{B}}:=\lim _{\rightarrow} B_{m}$.

Next, (28) together with [PT3, Lemma 2.2(iv)], implies that for any point $x=\left\{V_{k_{m}}\right\} \in$ $G O\left(k_{m}, V_{n_{m}}\right)$,

$$
B_{\tilde{m}}(x):=\left\{\mathbb{P}^{1} \in B_{\tilde{m}} \mid \mathbb{P}^{1} \ni x\right\} \simeq
$$

$\mathbb{P}\left(\left(\varphi_{\tilde{m}-1} \circ \ldots \circ \varphi_{m}\right)\left(V_{k_{m}}\right)^{*}\right) \times G O\left(1,\left(\varphi_{\tilde{m}-1} \circ \ldots \circ \varphi_{m}\right)\left(V_{k_{m}}\right)^{\perp} /\left(\varphi_{\tilde{m}-1} \circ \ldots \circ \varphi_{m}\right)\left(V_{k_{m}}\right)\right), \quad \tilde{m} \geq m$, and

$$
\Pi_{\tilde{m}}(x):=\left\{\left(\left(\varphi_{\tilde{m}-1} \circ \ldots \circ \varphi_{m}\right)\left(V_{k_{m}}\right), V_{k_{\tilde{m}+1}}\right) \in \Pi_{\tilde{m}}\right\} \simeq G O\left(1, V_{k_{\tilde{m}}}^{\perp} / V_{k_{\tilde{m}}}\right), \quad \tilde{m} \geq m .
$$

Since the quadrics $G O\left(1,\left(\varphi_{\tilde{m}-1} \circ \ldots \circ \varphi_{m}\right)\left(V_{k_{m}}\right)^{\perp} /\left(\varphi_{\tilde{m}-1} \circ \ldots \circ \varphi_{m}\right)\left(V_{k_{m}}\right)\right)$ are connected, (A.ii) follows from (28). Furthermore, as for each $\tilde{m} \geq 1$ the variety $G O\left(1, V_{k_{\tilde{m}}}^{\perp} / V_{k_{\tilde{m}}}\right)$ is a smooth quadric hypersurface in the projective space $\mathbb{P}^{n_{\tilde{m}}-2 k_{\tilde{m}}-1}$, (130) and (31) directly imply (A.iii) and (A.iv).

In the remaining cases the same argument goes through if one makes the following modifications.

If $\mathbf{X}=\mathrm{GS}(\infty, \infty)$, the formulas for $B_{m}, \Pi_{m}$ and $\Pi_{\tilde{m}}(x)$ are the same as (28), (29), (30) and (31) respectively, with $G O$ substituted by $G S$ (use [PT3, Lemma 2.5]). Note also that $G S\left(1, V_{k_{\tilde{m}}}^{\perp} / V_{k_{\tilde{m}}}\right)$ is isomorphic to the projective space $\mathbb{P}\left(V_{k_{\tilde{m}}}^{\perp} / V_{k_{\tilde{m}}}\right)$.

For $\mathbf{X}=\mathrm{GO}^{1}(\infty, 0)=\lim _{\rightarrow} G O\left(k_{m}, V_{2 k_{m}+1}\right)$ we first identify $G O\left(k_{m}, V_{2 k_{m}+1}\right)$ with an irreducible component $G O\left(k_{m}+1, V_{2 k_{m}+2}\right)^{*}$ of $G O\left(k_{m}+1, V_{2 k_{m}+2}\right)$ - see [PT3, Section 2.3]. Consequently, $\mathbf{X} \simeq \lim G O\left(k_{m}, V_{2 k_{m}}\right)^{*}$. Next, instead of (28)-(29) one has $B_{m} \simeq G O\left(k_{m}-2, V_{2 k_{m}}\right)$, $\Pi_{m} \simeq G O\left(k_{m}-1, V_{2 k_{m}}\right), m \geq 1$. Respectively, instead of (30)-(31) one has $B_{\tilde{m}}(x) \simeq$

\footnotetext{
${ }^{1}$ The reader can check that $\mathbf{G}(\infty)$ also satisfies the properties $\mathrm{L}, \mathrm{A}, \mathrm{T}$.
} 
$G\left(k_{\tilde{m}}-2,\left(\varphi_{\tilde{m}-1} \circ \ldots \circ \varphi_{m}\right)\left(V_{k_{m}}\right)\right)$ for $x=\left\{V_{k_{m}}\right\}$. The latter fact can be proved by an argument similar to that of $\left[\mathrm{PT} 3\right.$, Lemma 2.2]. In addition, $\Pi_{\tilde{m}}(x) \simeq \mathbb{P}\left(\left(\varphi_{\tilde{m}-1} \circ \ldots \circ \varphi_{m}\right)\left(V_{k_{m}}\right)^{*}\right)$, $\tilde{m} \geq m$.

For $\mathbf{X}=\mathbf{G S}(\infty, 0)=\lim G S\left(k_{m}, V_{2 k_{m}}\right)$ one can show that (28)-(29) can be replaced by: $B_{m} \simeq$ $G S\left(k_{m}-1, V_{2 k_{m}}\right), \Pi_{m} \simeq G S\left(k_{m}, V_{2 k_{m}}\right)$. Respectively, (30)-(31) for $x=\left\{V_{k_{m}}\right\} \in G S\left(k_{m}, V_{2 k_{m}}\right)$ can be replaced by $B_{\tilde{m}}(x) \simeq G\left(k_{\tilde{m}}-1,\left(\varphi_{\tilde{m}-1} \circ \ldots \circ \varphi_{m}\right)\left(V_{k_{m}}\right)\right)$, and $\Pi_{\tilde{m}}(x) \simeq\left\{\mathbb{P}\left(\left(\varphi_{\tilde{m}-1} \circ \ldots \circ\right.\right.\right.$ $\left.\left.\left.\varphi_{m}\right)\left(V_{k_{m}}\right)^{*}\right)\right\}$ is a point for $\tilde{m} \geq m$.

\section{Linear sections of $\mathbf{G}(\infty), \mathbf{G O}(\infty, \infty), \mathbf{G S}(\infty, \infty)$}

\subsection{Linear sections of finite-dimensional grassmannians.}

Let $G=G(k, V), G O(k, V), G S(k, V)$. Assume $1 \leq k<\operatorname{dim} V-1$ for $G=G(k, V)$, and $1 \leq k<\left[\frac{\operatorname{dim} V}{2}\right]$ for $G=G O(k, V), G S(k, V)$. Put $N:=\operatorname{dim} H^{0}\left(\mathcal{O}_{G}(1)\right)$ and $V_{N}:=H^{0}\left(\mathcal{O}_{G}(1)\right)^{*}$. We consider $G$ as a subvariety of $\mathbb{P}\left(V_{N}\right)$ via the Plücker embedding. For a given integer $c$, $1 \leq c \leq k-1$, set

$$
X:=G \cap \mathbb{P}(U),
$$

where $U \subset V_{N}$ is a subspace of codimension $c$. We call $X$ a linear section of $G$ of codimension c.

Note that there is a single family of maximal projective spaces of dimension $k$ on $G$ with base $\tilde{G}$, where $\tilde{G}=G(k+1, V)$ if $G=G(k, V)$, respectively, $\tilde{G}=G O(k+1, V)$ if $G=G O(k, V)$, and $\tilde{G}=G S(k+1, V)$ if $G=G S(k, V)$ (see [PT3, Lemmas 2.2(i) and 2.5(i)]). Consider the graph of incidence $\Sigma:=\left\{\left(V_{k}, V_{k+1}\right) \in G \times \tilde{G} \mid V_{k} \subset V_{k+1}\right\}$ with projections $\tilde{G} \stackrel{p}{\leftarrow} \Sigma \stackrel{q}{\rightarrow} G$ and set $\pi:=\left.p\right|_{q^{-1}(X)}: q^{-1}(X) \rightarrow \tilde{G}$. The condition $1 \leq c \leq k-1$ implies that $\pi$ is a surjective projective morphism.

Proposition 5.1. For a subspace $U \subset V_{N}$ of codimension $c$ in general position the following statements hold.

(i) The varieties $X$ and $q^{-1}(X)$ are smooth and

$$
\pi_{*} \mathcal{O}_{q^{-1}(X)}=\mathcal{O}_{\tilde{G}}
$$

(ii) $Z(U):=\left\{x \in \tilde{G} \mid \operatorname{dim} \pi^{-1}(x)>k-c\right\}$ is a proper closed subset of $\tilde{G}$ and

$$
\operatorname{codim}_{\tilde{G}} Z(U) \geq 3, \quad \operatorname{codim}_{q^{-1}(X)} \pi^{-1}(Z(U)) \geq 2 .
$$

(iii) The projection $\pi: q^{-1}(X) \backslash \pi^{-1}(Z(U)) \rightarrow \tilde{G} \backslash Z(U)$ is a projective $\mathbb{P}^{k-c}$-bundle.

Proof. We give the proof for the case $G=G O(k, V)$. The other cases are very similar and we leave them to the reader.

(i) Since the projective subspace $\mathbb{P}(U)$ is in general position in $\mathbb{P}\left(V_{N}\right)$, we have $\operatorname{codim}_{G} X=c$ and hence there is a Koszul resolution of the $\mathcal{O}_{G^{-}}$-sheaf $\mathcal{O}_{X}$

$$
0 \rightarrow \mathcal{O}_{G}(-c) \rightarrow \ldots \rightarrow\left(\begin{array}{c}
c \\
i
\end{array}\right) \mathcal{O}_{G}(-i) \rightarrow \ldots \rightarrow c \mathcal{O}_{G}(-i) \rightarrow \mathcal{O}_{G} \rightarrow \mathcal{O}_{X} \rightarrow 0
$$

The pullback of (34) under the projection $q$ is a $\mathcal{O}_{\Sigma}$-resolution of the sheaf $\mathcal{O}_{q^{-1}(X)}=\pi^{*} \mathcal{O}_{\tilde{G}}$ of the form

$$
0 \rightarrow \mathcal{L}_{c} \rightarrow \ldots \rightarrow \mathcal{L}_{1} \rightarrow \mathcal{O}_{\Sigma} \rightarrow \pi^{*} \mathcal{O}_{\tilde{G}} \rightarrow 0
$$

where $\mathcal{L}_{i}:=q^{*}\left(\left(\begin{array}{l}c \\ i\end{array}\right) \mathcal{O}_{G}(-i)\right), i=1, \ldots, c$.

For any $x \in \tilde{G}$ we have $p^{-1}(x) \simeq \mathbb{P}^{k}$, so the condition $c \leq k-1 \operatorname{implies} H^{j}\left(p^{-1}(x),\left.\mathcal{L}_{i}\right|_{p^{-1}(x)}\right) \simeq$ $H^{j}\left(\mathbb{P}^{k}, \mathcal{O}_{\mathbb{P}^{k}}(-i)\right)=0$ for $j \geq 0, i=1, \ldots, c$. Hence the Base-change Theorem [H1, Ch. III, 
Theorem 12.11] for the flat projective morphism $p$ shows that $R^{j} p_{*} \mathcal{L}_{i}=0$. In addition, by the same reason $R^{j} p_{*} \mathcal{O}_{\Sigma}=0, j>0$, and clearly $p_{*} \mathcal{O}_{\Sigma}=\mathcal{O}_{\tilde{G}}$. Therefore, applying the functor $R p_{*}$ to (35) we obtain (32).

(ii) We now prove (33). Fix an arbitrary point $V_{k+1} \in \tilde{G}$. Since $p^{-1}(x)=\mathbb{P}\left(V_{k+1}^{*}\right)$ (see [PT3, Lemma 2.2(i)]), there is an induced monomorphism

$$
0 \rightarrow V_{k+1}^{*} \rightarrow V_{N}
$$

Consider the varieties

$$
\Gamma_{i}:=\left\{\left(W, V_{k+1}\right) \in G\left(N-c, V_{N}\right) \times \tilde{G} \mid \operatorname{dim}\left(W \cap V_{k+1}^{*}\right) \geq k-c+i+2\right\}, \quad 0 \leq i \leq c-1,
$$

together with the natural projections

$$
G\left(N-c, V_{N}\right) \stackrel{p_{i}}{\leftarrow} \Gamma_{i} \stackrel{q_{i}}{\rightarrow} \tilde{G} .
$$

For an arbitrary $U \in G\left(N-c, V_{N}\right)$ denote

$$
Z_{i}(U):=q_{i}\left(p_{i}^{-1}(U)\right), \quad 0 \leq i \leq c-1 .
$$

By construction, $Z_{0}(U)=Z(U), Z_{i}(U)$ are closed subvarieties of $\tilde{G}$, and we have a filtration

$$
\emptyset=: Z_{c}(U) \subset Z_{c-1}(U) \subset \ldots \subset Z_{0}(U)=Z(U)
$$

such that $\left.Z_{i}(U)^{\prime}:=Z_{i}(U) \backslash Z_{i+1}(U)\right)$ are locally closed subvarieties of $\tilde{G}$. Consequently, $B_{i}(U)^{\prime}:=\pi^{-1}\left(Z_{i}(U)^{\prime}\right)$ are locally closed subvarieties of $q^{-1}(X)$. Moreover, $\left.\pi\right|_{B_{i}(U)^{\prime}}: B_{i}(U)^{\prime} \rightarrow$ $Z_{i}(U)^{\prime}$ is a $\mathbb{P}^{k+1-c+i}$-bundle, so that $\operatorname{dim} B_{i}(U)^{\prime}=\operatorname{dim} Z_{i}(U)^{\prime}+k+1-c+i$. Equivalently,

$$
\operatorname{codim}_{q^{-1}(X)} B_{i}(U)^{\prime}=\operatorname{codim}_{\tilde{G}} Z_{i}(U)^{\prime}-(i+1) .
$$

Note also that $Z(U)=\cup_{i=0}^{c-1} Z_{i}(U)^{\prime}$, hence

$$
\pi^{-1}(Z(U))=\pi^{-1}\left(\cup_{i=0}^{c-1} Z_{i}(U)^{\prime}\right)=\cup_{i=0}^{c-1} B_{i}(U)^{\prime} .
$$

We now calculate the dimensions of $Z_{i}(U)$ under the assumption that $U$ is in general positon. For this, let $Y:=q_{i}^{-1}(x)$ be the fibre of the projection $q_{i}$ over a point $x=V_{k+1} \in Z_{i}(U)$. Consider the variety $\tilde{Y}=\left\{\left(W, V_{k-c+i+2}\right) \in G\left(N-c, V_{N}\right) \times G\left(k-c+i+2, V_{k+1}^{*}\right) \mid W \supset\right.$ $\left.V_{k-c+i+2} \subset V_{k+1}^{*}\right\}$. The natural projection $\tilde{Y} \rightarrow G\left(k-c+i+2, V_{k+1}^{*}\right)$ is a fibration with the grassmannian $G\left(N-k-i-2, \mathbb{C}^{N-k-i-2+c}\right)$ as a fibre. On the other hand, one has a birational surjective morphism $\tilde{Y} \rightarrow Y,\left(W, V_{k-c+i+2}\right) \mapsto W$. Therefore, in view of (136) $) \operatorname{dim} Y=\operatorname{dim} \tilde{Y}=$ $\operatorname{dim} G\left(k-c+i+2, V_{k+1}^{*}\right)+\operatorname{dim} G\left(N-k-i-2, \mathbb{C}^{N-k-i-2+c}\right)=c N-c^{2}+(i+1)(c-k-i-2)$. As $q_{i}$ is surjective, this yields

$$
\operatorname{dim} \Gamma_{i}=\operatorname{dim} \tilde{G}+\operatorname{dim} Y=\operatorname{dim} \tilde{G}+c N-c^{2}+(i+1)(c-k-i-2) .
$$

Since $p_{i}$ is also surjective, for a point $U \in G\left(N-c, V_{N}\right)$ in general position we have $\operatorname{dim} Z_{i}(U)=$ $\operatorname{dim} \Gamma_{i}-\operatorname{dim} G\left(N-c, V_{N}\right)=\operatorname{dim} \tilde{G}-(i+1)(k+i+2-c)$, i.e.

$$
\operatorname{codim}_{\tilde{G}} Z_{i}(U)=(i+1)(k+i+2-c), \quad 0 \leq i \leq c-1 .
$$

This together with (38) implies $\operatorname{codim}_{q^{-1}(X)} B_{i}(U)^{\prime}=(i+1)(k+i+1-c), 0 \leq i \leq c-1$. Therefore, in view of (39) and the assumption $c \leq k-1$, we obtain

$$
\operatorname{codim}_{q^{-1}(X)} \pi^{-1}(Z(U))=\min _{0 \leq i \leq c-1} \operatorname{codim}_{q^{-1}(X)} B_{i}(U)^{\prime}=k+1-c \geq 2 .
$$

The inequality $\operatorname{codim}_{\tilde{G}} Z(U) \geq 3$ follows now from (38), and the proposition is proved.

Corollary 5.2. Under the assumptions of Proposition 5.1 , let $\mathcal{E}$ be a vector bundle on $q^{-1}(X)$ trivial along the fibres of the morphism $\pi: q^{-1}(X) \rightarrow \tilde{G}$. Then there is an isomorphism ev $: \pi^{*} \pi_{*} \mathcal{E} \stackrel{\simeq}{\rightarrow} \mathcal{E}$.

Proof. Apply Proposition 7.2 from the appendix to the morphism $\pi: q^{-1}(X) \rightarrow \tilde{G}$, the subvariety $Z(U)$ in $\tilde{G}$, and the vector bundle $\mathcal{E}$ on $q^{-1}(X)$. 
Lemma 5.3. Let $X=G \cap \mathbb{P}(U)$ be a linear section of $G$ of codimension c for $1 \leq c \leq(k-1) / 2$, and let $\mathbb{P}^{1}$ be a projective line on $\tilde{G}$. Then there exists a rational curve $C \subset q^{-1}(X)$ such that $\left.\pi\right|_{C}$ is an isomorphism of $C$ with $\mathbb{P}^{1}$, and $\left.q\right|_{C}$ is either an isomorphism or a constant map.

Proof. We only consider the case $G=G O(k, V)$. It is clear that

$$
\mathbb{P}^{1}=\left\{V_{k+1} \in \tilde{G} \mid V_{k} \subset V_{k+1} \subset V_{k+2}\right\}
$$

for a unique isotropic flag $V_{k} \subset V_{k+2}$ in $V$. If $V_{k} \in X$, we set $C:=\left\{\left(V_{k}, V_{k+1}\right) \in \Sigma \mid V_{k} \subset\right.$ $\left.V_{k+1} \subset V_{k+2}\right\}$. Then $\left.\pi\right|_{C}: C \rightarrow \mathbb{P}^{1}$ is an isomorphism and $q(C)$ equals the point $\left\{V_{k}\right\} \in G$.

Assume that $V_{k} \notin X$. It is straightforward to check that the intersection $q\left(p^{-1}\left(\mathbb{P}^{1}\right)\right) \cap \mathbb{P}^{N-2}$ for a hyperplane $\mathbb{P}^{N-2} \subset \mathbb{P}\left(V_{N}\right)$ such that $V_{k} \notin \mathbb{P}^{N-2}$, is isomorphic to the direct product $\mathbb{P}\left(V_{k}^{*}\right) \times$ $\mathbb{P}\left(V_{k+2} / V_{k}\right)$ imbedded by Segre in $\mathbb{P}^{N-2}$. Let $\mathbb{P}_{a}^{k-1}, \mathbb{P}_{b}^{k-1}$ be the fibres in $\mathbb{P}\left(V_{k}^{*}\right) \times \mathbb{P}\left(V_{k+2} / V_{k}\right)$ over two points $a, b \in \mathbb{P}\left(V_{k+2} / V_{k}\right)$. The projection $\mathrm{pr}_{1}: \mathbb{P}\left(V_{k}^{*}\right) \times \mathbb{P}\left(V_{k+2} / V_{k}\right) \rightarrow \mathbb{P}\left(V_{k}^{*}\right)$ induces an isomorphism $f: \mathbb{P}_{a}^{k-1} \stackrel{\sim}{\rightarrow} \mathbb{P}_{b}^{k-1}$.

Set $\mathbb{P}_{a}^{k-c-1}:=\mathbb{P}_{a}^{k-1} \cap \mathbb{P}(U), \mathbb{P}_{b}^{k-c-1}:=\mathbb{P}_{b}^{k-1} \cap \mathbb{P}(U)$. Since $1 \leq c \leq(k-1) / 2$, the intersection $\mathbb{P}_{b}^{k-c-1} \cap f\left(\mathbb{P}_{a}^{k-c-1}\right) \subset \mathbb{P}_{b}^{k-1}$ is nonempty. Consider a point $x$ in this latter intersection. By construction, the fibre $\mathbb{P}_{x}^{1}:=p r_{1}^{-1}(x)$ lies in $q\left(p^{-1}\left(\mathbb{P}^{1}\right)\right) \cap X$. Finally, the preimage of $\mathbb{P}_{x}^{1}$ in $p^{-1}\left(\mathbb{P}^{1}\right)$ is a rational curve $C$ as desired.

Proposition 5.4. Let $X$ be a linear section of $G$ of codimension c for $1 \leq c \leq(k-1) / 2$. Then a linearly trivial vector bundle $E$ on $X$ is trivial.

Proof. Consider the vector bundle $\mathcal{E}:=q^{*} E$ on $q^{-1}(X)$. Since $E$ is linearly trivial, for any $\left.x \in \tilde{G} \mathcal{E}\right|_{\pi^{-1}(x)}$ is a linearly trivial bundle on the projective space $\pi^{-1}(x)$. A well-known theorem [OSS, Ch. I, Theorem 3.2.1] implies that $\left.\mathcal{E}\right|_{\pi^{-1}(x)}$ is trivial. Therefore ev $: \pi^{*} \pi_{*} \mathcal{E} \rightarrow \mathcal{E}$ is an isomorphism by Corollary 5.2 .

Next, Lemma 5.3 allows us to conclude that $\pi_{*} \mathcal{E}$ is linearly trivial. Indeed, if $\mathbb{P}^{1} \subset \tilde{G}$ is a projective line and $C \subset q^{-1}(X)$ is a rational curve as in Lemma 5.3, then $\left.\left.\pi_{*} \mathcal{E}\right|_{\mathbb{P}^{1}} \simeq \mathcal{E}\right|_{C}$, and hence $\left.\mathcal{E}\right|_{C}$ is trivial because of the linear triviality of $E$.

Consequently, $\pi_{*} \mathcal{E}$ is trivial by Proposition 7.4. from the appendix. Then $\mathcal{E} \simeq \pi^{*} \pi_{*} \mathcal{E}$ is also trivial. Finally, since $q: q^{-1}(X) \rightarrow X$ is a flat projective morphism with irreducible fibres, $E=q_{*} \mathcal{E}$ is trivial by Proposition 7.1 ,

\subsection{Linear sections of $\mathrm{G}(\infty), \mathrm{GO}(\infty, \infty), \mathrm{GS}(\infty, \infty)$ of small codimension.}

Let $\mathbf{G}=\mathbf{G}(\infty), \mathbf{G O}(\infty, \infty), \mathbf{G S}(\infty, \infty)$, in particular $\mathbf{G}=\lim _{\rightarrow} G\left(k_{m}, V_{n_{m}}\right), \lim _{\rightarrow} G O\left(k_{m}, V_{n_{m}}\right)$, $\lim _{\rightarrow} G S\left(k_{m}, V_{n_{m}}\right)$, see Definition 4.1, Fix a nondecreasing sequence $\left\{c_{m}\right\}_{m \geq 1}$ of integers satisfying the condition

$$
1 \leq c_{m} \leq\left(k_{m}-1\right) / 2 \text {. }
$$

Consider an ind-variety $\mathbf{X}=\lim _{\rightarrow} X_{m}$ such that, for each $m \geq 1, X_{m}$ is a smooth linear section of codimension $c_{m}$ of $G_{m}=G\left(k_{m}, V_{n_{m}}\right), G O\left(k_{m}, V_{n_{m}}\right), G S\left(k_{m}, V_{n_{m}}\right)$ and the embedding $\varphi_{m}$ : $X_{m} \hookrightarrow X_{m+1}$ is induced by the embedding $G_{m} \hookrightarrow G_{m+1}$. In what follows we call such indvarieties linear sections of $\mathbf{G}$ of small codimension.

The existence of linear sections $\mathbf{X}$ of $\mathbf{G}$ of small codimension is a consequence of the Bertini Theorem. Moreover, such a linear section $\mathbf{X}$ is a linear ind-variety and $\mathrm{Pic} \mathbf{X}$ is generated by $\mathcal{O}_{\mathbf{X}}(1):=\lim _{\leftarrow} \mathcal{O}_{X_{m}}(1)$. This follows from the observation that $\mathcal{O}_{X_{m}}(1)$ generates Pic $X_{m}$ by the Lefschetz Theorem, and from the linearity of the embeddings $G_{m} \hookrightarrow G_{m+1}$.

Proposition 5.5. Let $\mathbf{G}=\mathbf{G}(\infty), \mathbf{G O}(\infty, \infty), \mathbf{G S}(\infty, \infty)$. There exists a linear section $\mathbf{X}$ of small codimension of $\mathbf{G}$ which is not isomorphic to either of the ind-varieties $\mathbf{G}(\infty)$, $\mathrm{GO}(\infty, \infty)$ or $\mathbf{G S}(\infty, \infty)$. 
Proof. Let $\mathbf{G}=\lim _{\rightarrow} G_{m}$ where $G_{m}=G\left(k_{m}, V_{n_{m}}\right), G O\left(k_{m}, V_{2 n_{m}+1}\right), G S\left(k_{m}, V_{2 n_{m}}\right)$. Fix $m \geq 1$ and let $\mathbb{P}^{1}$ be a projective line on $G_{m}$. Then there exist unique maximal projective subspaces $\mathbb{P}^{k_{m}}$ and $\mathbb{P}^{s_{m}}$ on $G_{m}$ which intersect in $\mathbb{P}^{1}$. For $G_{m}=G\left(k_{m}, V_{n_{m}}\right)$ one has $s_{m}=n_{m}-k_{m}$, and for $G_{m}=G O\left(k_{m}, V_{n_{m}}\right), G S\left(k_{m}, V_{n_{m}}\right)$ one has $s_{m}=\left[\frac{n_{m}}{2}\right]-k_{m}$, see [PT3, Lemmas 2.3(iii) and 2.6(ii)].

For $\tilde{m} \geq m$ the embeddings $\varphi_{\tilde{m}}: G_{\tilde{m}} \hookrightarrow G_{\tilde{m}+1}$ in the direct limit $\lim _{\rightarrow} G_{m}$ are given by formula (24), which makes it easy to check that $\mathbb{P}^{k_{m}}$ and $\mathbb{P}^{s_{m}}$ admit extensions $\mathbb{P}^{k_{\tilde{m}}} \subset G_{\tilde{m}}, \mathbb{P}^{s_{\tilde{m}}} \subset G_{\tilde{m}}$ such that $\mathbb{P}^{k_{m}} \subset \mathbb{P}^{k_{\tilde{m}}}, \mathbb{P}^{s_{m}} \subset \mathbb{P}^{s_{\tilde{m}}}$ and

$$
\mathbb{P}^{1}=\mathbb{P}^{k_{\tilde{m}}} \cap \mathbb{P}^{s_{\tilde{m}}}, \quad \tilde{m} \geq m .
$$

Denoting $\mathbf{P}_{\alpha}^{\infty}:=\lim _{\rightarrow} \mathbb{P}^{k_{\tilde{m}}}, \mathbf{P}_{\beta}^{\infty}:=\lim _{\rightarrow} \mathbb{P}^{s_{\tilde{m}}}$, we have

$$
\mathbb{P}^{1}=\mathbf{P}_{\alpha}^{\infty} \cap \mathbf{P}_{\beta}^{\infty} .
$$

We now choose $n_{\tilde{m}}$ and $k_{\tilde{m}}$ in a specific way. Namely, we assume that $n_{\tilde{m}}=3 t_{\tilde{m}}$ for $G_{m}=$ $G\left(k_{m}, V_{n_{m}}\right)$, and $\left[\frac{n_{\tilde{m}}}{2}\right]=3 t_{\tilde{m}}$ for $G_{m}=G O\left(k_{m}, V_{n_{m}}\right), G S\left(k_{m}, V_{n_{m}}\right), k_{\tilde{m}}=2 t_{\tilde{m}}, t_{\tilde{m}} \in \mathbb{Z}_{\geq 1}$. Set $c_{\tilde{m}}:=t_{\tilde{m}}-1$. Then

$$
s_{\tilde{m}}-c_{\tilde{m}}=1
$$

and the inequality (42) together with the conditions $\lim _{\tilde{m} \rightarrow \infty} k_{\tilde{m}}=\lim _{\tilde{m} \rightarrow \infty} s_{\tilde{m}}=\infty$ are satisfied. Next, using (44) and the Bertini Theorem we choose a tower of projective subspaces $\mathbb{P}^{N_{\tilde{m}}-c_{\tilde{m}}-1} \subset$ $\mathbb{P}\left(V_{N_{\tilde{m}}}\right)$ for $\tilde{m} \geq m$ in general positon so that $\mathbb{P}^{N_{\tilde{m}}-c_{\tilde{m}}-1} \cap \mathbb{P}^{s_{\tilde{m}}}=\mathbb{P}^{1}$ and $\mathbb{P}^{N_{\tilde{m}}-c_{\tilde{m}}-1} \cap \mathbb{P}^{k_{\tilde{m}}}=$ $\mathbb{P}^{\left(k_{\tilde{m}} / 2\right)+1}$ for some projective subspaces $\mathbb{P}^{\left(k_{\tilde{m}} / 2\right)+1}$ of $G_{\tilde{m}}$. As a result, we obtain a linear section $\mathbf{X}:=\lim _{\rightarrow}\left(\mathbb{P}^{N_{\tilde{m}}-c_{\tilde{m}}-1} \cap G_{\tilde{m}}\right)$ of $\mathbf{G}$ of small codimension and a projective line $\mathbb{P}^{1} \subset \mathbf{X}$ such that $\mathbb{P}^{1}$ is contained in a unique linear ind-projective subspace $\mathbf{P}^{\infty}$ of $\mathbf{X}$, namely $\mathbf{P}^{\infty}:=\lim \mathbb{P}^{\left(k_{\tilde{m}} / 2\right)+1}$. If $\mathbf{X}$ were isomorphic to $\mathbf{G}(\infty), \mathbf{G O}(\infty, \infty), \mathbf{G S}(\infty, \infty)$, this would contradict to (43)).

Now we show that a linear section $\mathbf{X}$ of $\mathbf{G}$ of small codimension satisfies the properties L, A and $\mathrm{T}$. The property $\mathrm{L}$ is clear as $\mathrm{Pic} \mathbf{X}$ is generated by $\mathcal{O}_{\mathbf{X}}(1)$, and $H^{1}\left(X_{m}, \mathcal{O}_{X_{m}}(a)\right)$ vanishes for $a<0$ by Kodaira's Theorem. The property $\mathrm{T}$ is established in Proposition 5.4. It remains to establish the property A. Part (A.i) is clear as $\mathcal{O}_{X_{m}}(1)$ is very ample. For parts (A.ii)-(A.iv) we consider in detail only the case when $\mathbf{X}$ is a linear section of $\mathbf{G O}(\infty, \infty)$.

Let $B\left(G_{m}\right)$ be the family of all projective lines in $G_{m}=G O\left(k_{m}, V_{n_{m}}\right)$, and $B_{m}$ be its subfamily consisting of those projective lines which lie in $X_{m}$. By definition, $X_{m}$ is the intersection of $G_{m}$ with a subspace $\mathbb{P}\left(U_{m}\right)$ of $\mathbb{P}\left(V_{N_{m}}\right)$ for a fixed $U_{m} \in G\left(N_{m}-c_{m}, V_{N_{m}}\right)$ in general position. The grassmannians $G\left(2, V_{N_{m}}\right)$ and $G\left(2, U_{m}\right)$ can be be thought of as the grassmannians of projective lines in $\mathbb{P}\left(V_{N_{m}}\right)$ and $\mathbb{P}\left(U_{m}\right)$ respectively. Then $B_{m}=B\left(G_{m}\right) \cap G\left(2, U_{m}\right)$ where the intersection is taken in $G\left(2, V_{N_{m}}\right)$. We show next that $B_{m}$ is irreducible.

Let $B$ be an irreducible component of $B_{m}$. Since $G\left(2, V_{N_{m}}\right)$ is smooth, the subadditivity of codimensions [Ha, Thm. 17.24]) yields

$$
\operatorname{codim}_{B\left(G_{m}\right)} B \leq \operatorname{codim}_{G\left(2, V_{N_{m}}\right)} G\left(2, U_{m}\right)=2 c_{m} .
$$

Consider the graph of incidence $\Sigma_{m}:=\left\{\left(x, \mathbb{P}^{k_{m}}\right) \in G_{m} \times \tilde{G}_{m} \mid x \in \mathbb{P}^{k_{m}}\right\}$ with its projections $\tilde{G}_{m} \stackrel{p_{m}}{\leftarrow} \Sigma_{m} \stackrel{q_{m}}{\rightarrow} G_{m}$, where $\tilde{G}_{m}:=G O\left(k_{m}+1, V_{n_{m}}\right)$. Let $B\left(\Sigma_{m}\right)$ be the family of all projective lines in $\Sigma_{m}$ lying in the fibres of the projection $p_{m}$. Denote by $B\left(q_{m}^{-1}\left(X_{m}\right)\right)$ the subfamily of $B\left(\Sigma_{m}\right)$ consisting of those projective lines which lie in $q_{m}^{-1}\left(X_{m}\right)$. The projection $q_{m}: \Sigma_{m} \rightarrow G_{m}$ induces a morphism $r_{G_{m}}: B\left(\Sigma_{m}\right) \rightarrow B\left(G_{m}\right)$ which is bijective since any projective line on $G_{m}$ lies in a unique maximal projective space $\mathbb{P}^{k_{m}}$ (see [PT3, Section 2]). The space $\mathbb{P}^{k_{m}}$ is an isomorphic image via $q_{m}$ of some fibre of $p_{m}$. Respectively, the restricted morphism 
$r_{X_{m}}:=\left.r_{G_{m}}\right|_{B\left(q_{m}^{-1}\left(X_{m}\right)\right)}: B\left(q_{m}^{-1}\left(X_{m}\right)\right) \rightarrow B_{m}$ is a bijection. Hence, for any irreducible component $B^{\prime}$ of $B\left(q_{m}^{-1}\left(X_{m}\right)\right)$, (45) yields the inequality

$$
\operatorname{codim}_{B\left(\Sigma_{m}\right)} B^{\prime} \leq \operatorname{codim}_{G\left(2, V_{N_{m}}\right)} G\left(2, U_{m}\right)=2 c_{m} .
$$

The projection $p_{m}$ induces a projection $\rho_{m}: B\left(\Sigma_{m}\right) \rightarrow \tilde{G}_{m}$. Let

$$
\emptyset=Z_{c_{m}}\left(U_{m}\right) \subset Z_{c_{m}-1}\left(U_{m}\right) \subset \ldots \subset Z_{0}\left(U_{m}\right) \subset \tilde{G}_{m}
$$

be the filtration (37) of $\tilde{G}_{m}$ by closed subvarieties $Z_{i}\left(U_{m}\right)$ of codimensions in $\tilde{G}_{m}$ given by (40) where we put $c=c_{m}, k=k_{m}$. This filtration yields a decomposition $B\left(q_{m}^{-1}\left(X_{m}\right)\right)=\underset{0 \leq i \leq c_{m}}{\sqcup} B_{i}$, where $B_{0}:=B\left(q_{m}^{-1}\left(X_{m}\right)\right) \cap \rho_{m}^{-1}\left(\tilde{G}_{m} \backslash Z_{0}\left(U_{m}\right)\right), B_{i}:=B\left(q_{m}^{-1}\left(X_{m}\right)\right) \cap \rho_{m}^{-1}\left(Z_{i-1}\left(U_{m}\right) \backslash Z_{i}\left(U_{m}\right)\right), i=$ $1, \ldots, c_{m}$. Formula (40) implies that $\operatorname{codim}_{B\left(\Sigma_{m}\right)} B_{0}=2 c_{m}, \operatorname{codim}_{B\left(\Sigma_{m}\right)} B_{i}>2 c_{m}$ for $i=1, \ldots, c_{m}$. This together with (46) yields the irreducibility of $B\left(q_{m}^{-1}\left(X_{m}\right)\right)$, hence of $B_{m}$ as well.

Now let $\Pi\left(G_{m}\right)$ be the family of projective spaces $\mathbb{P}^{k_{m}-1}$ lying in $B\left(G_{m}\right)$ and defined by the right-hand side of (29). Set

$$
\Pi:=\lim _{\rightarrow} \Pi_{m}
$$

where $\Pi_{m}:=\left\{\mathbb{P}^{k_{m}-c_{m}-1} \subset B_{m} \mid \mathbb{P}^{k_{m}-c_{m}-1}\right.$ is a linear subspace of some $\left.\mathbb{P}^{k_{m}-1} \in \Pi\left(G_{m}\right)\right\}$.

Fix $m$ and let $\pi:=\left.p_{m}\right|_{q_{m}^{-1}\left(X_{m}\right)}: q_{m}^{-1}\left(X_{m}\right) \rightarrow \tilde{G}_{m}$ be the projection. Consider the relative grassmannian $G_{\pi}:=\left\{\mathbb{P}^{1} \subset q_{m}^{-1}\left(X_{m}\right) \mid \mathbb{P}^{1}\right.$ lies linearly in a fibre of the projection $\left.\pi\right\}$ with induced projections $\rho: G_{\pi} \rightarrow \tilde{G}_{m}$ and $q_{\pi}: G_{\pi} \rightarrow B_{m}$. By definition, the fibre $\rho^{-1}(x)$ over an arbitrary point $x \in \tilde{G}_{m}$ is the grassmannian of projective lines in the projective space $\pi^{-1}(x)$. (Note that for a point $x \in \tilde{G}_{m}$ in general position the fibre $\pi^{-1}(x)$ is a projective space $\mathbb{P}^{k_{m}-c_{m}}$, hence $\rho^{-1}(x) \simeq G\left(2, \mathbb{C}^{k_{m}-c_{m}+1}\right)$.) Furthermore, the projection $q_{\pi}$ is birational.

By construction, the projective spaces $\mathbb{P}^{k_{m}-c_{m}-1} \in \Pi_{m}$ are isomorphic images under $q_{\pi}$ of projective spaces $\mathbb{P}^{k_{m}-c_{m}-1}$ lying linearly in the fibres of $\rho: G_{\pi} \rightarrow \tilde{G}_{m}$. Considering the set $G_{\rho}:=\left\{\mathbb{P}^{k_{m}-c_{m}} \subset q_{m}^{-1}\left(X_{m}\right) \mid \mathbb{P}^{k_{m}-c_{m}}\right.$ lies linearly in a fibre of $\left.\pi: q_{m}^{-1}\left(X_{m}\right) \rightarrow \tilde{G}_{m}\right\}$, we obtain a $\mathbb{P}^{k_{m}-c_{m}}$-fibration $q_{\rho}: \Pi_{m} \rightarrow G_{\rho}$ with fibre $q_{\rho}^{-1}(y)=\mathbb{P}^{k_{m}-c_{m}}$ over a given point $y=\left\{\mathbb{P}^{k_{m}-c_{m}}\right\} \in G_{\rho}$. Trerefore, to check the irreducibility of $\Pi_{m}$ it suffices to check the irreducibility of $G_{\rho}$. Note that the projection $\pi$ induces a projection $\tau: G_{\rho} \rightarrow \tilde{G}_{m}$ such that the fibre of $\tau$ over a point $V_{k_{m}+1} \in \tilde{G}_{m}$ coincides with the grassmannian $G\left(k_{m}-c_{m}+1, W\right)$, where $W \subset V_{k_{m}+1}$ is the subspace defined by the condition $\mathbb{P}(W)=\pi^{-1}(x)$. As above, (40) implies that, for $i \geq 1$, the locally closed subsets $\tau^{-1}\left(Z_{i}(W)\right)$ of $G_{\rho}$ have dimensions strictly less than that of the open subset $\tau^{-1}\left(Z(W) \backslash Z_{i}(W)\right)$. This proves the irreducibility of $G_{\rho}$, hence of $\Pi_{m}$.

Next, (30) implies that, for any point $x=V_{k_{m}} \in X_{m}$ and $\tilde{m} \geq m$, the base $B_{m}(x)$ of the family of projective lines on $X_{m}$ passing through $x$ is a linear section of the variety $\mathbb{P}\left(\left(\varphi_{\tilde{m}-1} \circ\right.\right.$ $\left.\left.\ldots \circ \varphi_{m}\right)\left(V_{k_{m}}\right)^{*}\right) \times G O\left(1,\left(\varphi_{\tilde{m}-1} \circ \ldots \circ \varphi_{m}\right)\left(V_{k_{m}}\right)^{\perp} /\left(\varphi_{\tilde{m}-1} \circ \ldots \circ \varphi_{m}\right)\left(V_{k_{m}}\right)\right) \subset \mathbb{P}\left(V_{k_{\tilde{m}}}^{*} \otimes V_{k_{\tilde{m}}}^{\perp} / V_{k_{\tilde{m}}}\right)$ by a projective subspace of codimension $c_{\tilde{m}}$ in $\mathbb{P}\left(V_{k_{\tilde{m}}}^{*} \otimes V_{k_{\tilde{m}}}^{\perp} / V_{k_{\tilde{m}}}\right)$. Let $b_{\tilde{m}}(x): B_{\tilde{m}}(x) \rightarrow Q_{(\tilde{m})}(x):=$ $G O\left(1, V_{k_{\tilde{m}}}^{\perp} / V_{k_{\tilde{m}}}\right)$ be the natural projection. Note that the fibres of $b_{\tilde{m}}(x)$ are projective spaces of dimension at least $k_{\tilde{m}}-c_{\tilde{m}}-1$, and, for points $x$ of $X_{m}$ and $z \in Q_{\tilde{m}}(x)$ in general position the fibre $b_{\tilde{m}}(x)^{-1}(z)$ is a projective space $\mathbb{P}^{k_{\tilde{m}}-c_{\tilde{m}}-1}$ by the Bertini Theorem. Moreover, we have an ind-variety $\mathbf{B}(x)=\lim _{\rightarrow} B_{\tilde{m}}(x), \tilde{m} \geq m$.

In a similar way we obtain that $\Pi(x):=\left\{\mathbf{P}^{\infty} \in \boldsymbol{\Pi} \mid \mathbf{P}^{\infty} \ni x\right\}$ is the ind-variety $\lim _{\tilde{m}}(x)$, $\tilde{m} \geq m$, where $\Pi_{\tilde{m}}(x):=\left\{\mathbb{P}^{k_{\tilde{m}}-c_{\tilde{m}}-1} \subset B_{m} \mid \mathbb{P}^{k_{\tilde{m}}-c_{\tilde{m}}-1}\right.$ lies as a linear projective subspace in a fibre of the projection $\left.b_{\tilde{m}}(x)\right\}$. Let $p(x): \Pi_{\tilde{m}}(x) \rightarrow Q_{(\tilde{m})}(x)$ be the induced projection. By construction, for any point $z \in Q_{(\tilde{m})}(x)$ the fibre $p(x)^{-1}(z)$ is the grassmannian $G\left(k_{\tilde{m}}-\right.$ $c_{\tilde{m}}, \mathbb{C}^{\operatorname{dim}\left(b_{\tilde{m}}(x)^{-1}(z)\right)+1}$ ). (In particular, this grassmannian is just a point for $x \in X_{\tilde{m}}$ and $z \in$ 
$Q_{(\tilde{m})}(x)$ in general position.) This implies the property (A.ii) since $Q_{(\tilde{m})}(x)$ is an irreducible quadric hypersurface.

The property (A.iii) is evident. As for the property (A.iv), let $Y$ be a fixed variety and $f: \Pi_{\tilde{m}}(x) \rightarrow Y$ be a morphism. For $\tilde{m} \rightarrow \infty$ the fibres of $p(x)$ are either points or are grassmannians whose dimensions tend to infinity. Therefore $f$ maps each fibre of $p(x)$ to a point, i.e. $f$ factors through the induced morphism $g: Q_{(\tilde{m})}(x) \rightarrow Y$. As $Q_{(\tilde{m})}(x)$ is a smooth quadric hypersurface whose dimension tends to infinity as $\tilde{m} \rightarrow \infty$, it follows that for large enough $\tilde{m}$ the morphism $g$ is a constant map. Hence, $f$ is constant too, and (A.iv) is proved.

Theorem 2.1 yields now the following.

Theorem 5.6. A vector bundle on a linear section $\mathbf{X}$ of small codimension of $\mathbf{G}(\infty)$, $\mathrm{GO}(\infty, \infty), \mathbf{G S}(\infty, \infty)$ is isomorphic to a direct sum of line bundles $\mathcal{O}_{\mathbf{X}}\left(a_{i}\right)$ for $a_{i} \in \mathbb{Z}$.

\section{Ind-PRoducts AND their subVarieties SATISFYing the PROPERTIEs L, A, T}

\subsection{Finite or countable ind-products satisfying the properties $\mathrm{L}, \mathrm{A}, \mathrm{T}$.}

Let $\mathbf{X}^{\xi}=\lim X_{m}^{\xi}, \xi \in \Xi$, be a countable collection of ind-varieties. We assume that for each $\xi \in \Xi$ and each $m \geq 1$ we have a fixed inclusion $X_{m}^{\xi} \subset X_{m+1}^{\xi}$. On every $\mathbf{X}^{\xi}$ we fix a point $x_{0}^{\xi}$. Without loss of generality we assume that $x_{0}^{\xi} \in X_{1}^{\xi}$. Fix a bijection $\nu: \mathbb{N} \stackrel{\sim}{\rightarrow} \Xi$ and denote $\underline{m}:=\{1,2, \ldots, m\}$. Set

$$
{ }_{\nu} X_{m}:=\underset{\xi \in \nu(\underline{m})}{\times} X_{m}^{\xi}
$$

and consider the embeddings

$$
{ }_{\nu} X_{m} \hookrightarrow{ }_{\nu} X_{m+1}={ }_{\nu} X_{m} \times X_{m}^{\nu(m+1)}, \quad x \mapsto\left(x, x_{0}^{\nu(m+1)}\right), m \geq 1 .
$$

We call the ind-variety $\mathbf{X}:=\lim _{\rightarrow} X_{m}$ an ind-product of the ind-varieties $\left\{\mathbf{X}^{\xi}\right\}_{\xi \in \Xi}$ and denote it as

$$
\mathbf{X}=\underset{\xi \in \Xi}{\times} \mathbf{X}^{\xi}
$$

Note that $\mathbf{X}$ does not depend, up to an isomorphism of ind-varieties, on the choice of the bijection $\nu: \mathbb{N} \stackrel{\sim}{\rightarrow} \Xi$, and thus the notation (48) is consistent. Indeed, let $\nu^{\prime}: \mathbb{N} \stackrel{\sim}{\rightarrow} \Xi$ be another bijection, and let $\psi:=\nu^{\prime-1} \circ \nu: \mathbb{N} \stackrel{\sim}{\rightarrow} \mathbb{N}$ be the induced bijection. An isomorphism $\mathbf{f}: \lim _{\nu} X_{m} \stackrel{\sim}{\rightarrow} \lim _{\nu^{\prime}} X_{m}, \mathbf{f}=\left\{f_{m}:{ }_{\nu} X_{m} \rightarrow{ }_{\nu^{\prime}} X_{\tilde{m}(m)}\right\}$, and its inverse $\mathbf{g}=\mathbf{f}^{-1}: \lim _{\nu^{\prime}} X_{m} \stackrel{\sim}{\rightarrow}$ $\lim _{\nu} X_{m}, \mathbf{g}=\left\{g_{m}:{ }_{\nu^{\prime}} X_{m} \rightarrow{ }_{\nu} X_{\tilde{m}(m)}\right\}$, for $\tilde{m}(m):=\min _{m^{\prime}>m}\left\{m^{\prime} \mid \psi(\underline{m}) \subset \underline{m}^{\prime}\right\}$, are given by the formulas

$$
\begin{aligned}
f_{m}:{ }_{\nu} X_{m}=\underset{\xi \in \nu(\underline{m})}{\times} X_{m}^{\xi} & \rightarrow{ }_{\nu^{\prime}} X_{\tilde{m}(m)}=\left(\underset{\xi \in \nu(\underline{m})}{\times} X_{\tilde{m}(m)}^{\xi}\right) \times\left(\underset{\xi \in \nu^{\prime}(\underline{\tilde{m}(m)}) \backslash \nu(\underline{m})}{\times} X_{\tilde{m}(m)}^{\xi}\right), \\
& x \mapsto\left(x,\left\{x_{0}^{\xi}\right\}_{\xi \in \nu^{\prime}(\tilde{\tilde{m}(m))}) \backslash \nu(\underline{m})}\right), \\
g_{m}: \nu^{\prime} X_{m}=\underset{\xi \in \nu^{\prime}(\underline{m})}{\times} X_{m}^{\xi} & \rightarrow{ }_{\nu} X_{\tilde{m}(m)}=\left(\underset{\xi \in \nu^{\prime}(\underline{m})}{\times} X_{\tilde{m}(m)}^{\xi}\right) \times\left(\underset{\xi \in \nu(\underline{\tilde{m}(m)}) \backslash \nu^{\prime}(\underline{m})}{\times} X_{\tilde{m}(m)}^{\xi}\right), \\
x & \mapsto\left(x,\left\{x_{0}^{\xi}\right\}_{\xi \in \nu(\underline{\tilde{m}(m))}) \backslash \nu^{\prime}(\underline{m})}\right) .
\end{aligned}
$$

Note in addition that in principle $\mathbf{X}$ depends on the choice of points $x_{0}^{\xi}$, however we suppress this dependence in the notation (48).

The reason we call $\mathbf{X}$ an ind-product rather than a product is that $\mathbf{X}$ is not a direct product in the category of ind-varieties. Of course, there are well-defined projections of ind-varieties $\mathbf{p}_{\xi}: \mathbf{X} \rightarrow \mathbf{X}^{\xi}, \mathbf{p}_{\xi}=\lim _{\rightarrow} p_{\xi m}$, where $p_{\xi m}:{ }_{\nu} X_{m} \rightarrow X_{m}^{\xi}$ is the projection onto the $\xi$-factor for 
$\xi \in \nu(\underline{m})$, and the constant map $p_{\xi m}:{ }_{\nu} X_{m} \rightarrow x_{0}^{\nu(m)}$ for $\xi \notin \nu(\underline{m})$. However, $\mathbf{X}$ fails to satisfy the universality property of a product.

If $\Xi$ is finite, we define the ind-product $\underset{\xi \in \Xi}{\times \mathbf{X}^{\xi}}$ as the set-theoretic direct product of the $\mathbf{X}^{\xi}$ 's. Then $\underset{\xi \in \Xi}{\times} \mathbf{X}^{\xi}=\lim _{\rightarrow}\left(\underset{\xi \in \Xi}{\times} X_{m}^{\xi}\right)$, and $\underset{\xi \in \Xi}{\times} \mathbf{X}^{\xi}$ clearly satisfies the universality property of a direct product in the category of ind-varieties.

Now let $\Xi$ be finite or countable and let the ind-varieties $\mathbf{X}^{\xi}=\lim _{\rightarrow} X_{m}^{\xi}$ satisfy the properties L, A and T. This means that on each $\mathbf{X}^{\xi}$ there exists a collection $\mathbf{L}^{\xi}:=\left\{\mathbf{L}_{\theta}^{\xi} \mid \theta \in \Theta_{\mathbf{X}^{\xi}}\right\}$ of line bundles and a collection $\mathbf{B}^{\xi}:=\left\{\mathbf{B}_{\theta}^{\xi}=\lim _{\rightarrow} B_{\theta m}^{\xi} \mid \theta \in \Theta_{\mathbf{X}^{\xi}}\right\}$ of ind-varieties of projective lines such that $\mathbf{X}^{\xi}$ satisfies the properties L, $\vec{A}$ and T. The collections $\left\{\mathbf{L}^{\xi}\right\}_{\xi \in \Xi \text { yield the following }}$ countable collection of line bundles on $\mathbf{X}$

$$
\mathbf{L}=\left\{\mathbf{p}_{\xi}^{*} \mathbf{L}_{\theta}^{\xi} \mid \theta \in \Theta_{\mathbf{X}^{\xi}}, \xi \in \Xi\right\} .
$$

Moreover, any projective line $\mathbb{P}^{1}$ on $\mathbf{X}^{\xi}$ determines a projective line $\mathbb{P}_{\mathbf{X}}^{1}$ on $\mathbf{X}$ such that $\mathbf{p}_{\xi}\left(\mathbb{P}_{\mathbf{X}}^{1}\right)=$ $\mathbb{P}^{1}$ and $\mathbf{p}_{\xi^{\prime}}\left(\mathbb{P}_{\mathbf{X}}^{1}\right)=\left\{x_{0}^{\xi^{\prime}}\right\}$ for $\xi^{\prime} \neq \xi$. Therefore each ind-variety $\mathbf{B}_{\theta}^{\xi}$ of projective lines on $\mathbf{X}^{\xi}$ "lifts" to an ind-variety of projective lines on $\mathbf{X}$. In this way we obtain a collection $\mathbf{B}$ of indvarieties of projective lines on $\mathbf{X}$. Since each $\mathbf{X}^{\xi}$ satisfies the properties L, A and T, it is easy to check that $\mathbf{X}$ satisfies the same properties with respect to the collections $\mathbf{L}$ and $\mathbf{B}$.

This, together with Theorem 2.1, leads to the following theorem.

Theorem 6.1. A vector bundle on $\mathbf{X}=\underset{\xi \in \Xi}{\times \mathbf{X}^{\xi}}$, where each ind-variety $\mathbf{X}^{\xi}$ satisfies the properties $L, A$ and $T$, is isomorphic to a direct sum of line bundles.

\subsection{Linear sections of ind-products.}

In this subsection we assume that $\Xi$ is finite, $\Xi=\{1,2, \ldots, l\}$, and that the ind-varieties $\mathbf{X}^{i}=$ $\lim _{\rightarrow} X_{m}^{i}, i \in \Xi$, are copies of the standard ind-grassmannians $\mathbf{G}(\infty), \mathbf{G O}(\infty, \infty), \mathbf{G S}(\infty, \infty)$. By the above, $\underset{i \in \Xi}{\times} \mathbf{X}^{i}$ is a direct $\operatorname{limit} \underset{\rightarrow}{\lim }\left(\underset{i \in \Xi}{\times} X_{m}^{i}\right)$. Each $X_{m}^{i}$ is a (possibly isotropic) grassmannian which we consider as lying via the Plücker embedding in $\mathbb{P}^{N_{i m}-1}=\mathbb{P}\left(V_{N_{i m}}\right)$, and the embeddings $\underset{i \in \Xi}{\times} X_{m}^{i} \hookrightarrow \underset{i \in \Xi}{\times} X_{m+1}^{i}$ are induced by standard extensions $X_{m}^{i} \hookrightarrow X_{m+1}^{i}$. We also assume that $\underset{i \in \Xi}{\times} X_{m}^{i}$ lies in $\mathbb{P}^{N_{m}-1}$ via the Segre embedding $\underset{i \in \Xi}{\times} \mathbb{P}^{N_{i m}-1} \hookrightarrow \mathbb{P}^{N_{m}-1}$.

For each $m \geq 1$ and $i \in \Xi$ set

$$
\widehat{X}_{m}^{i}:=X_{m}^{1} \times \ldots \times X_{m}^{i-1} \times X_{m}^{i+1} \times \ldots \times X_{m}^{l},
$$

and for $X_{m}^{i}=G\left(k_{i m}, V_{n_{i m}}\right)$ (respectively, $X_{m}^{i}=G O\left(k_{i m}, V_{n_{i m}}\right)$ or $X_{m}^{i}=G S\left(k_{i m}, V_{n_{i m}}\right)$ ), set $X_{m}^{i}{ }^{+}:=G\left(k_{i m}+1, V_{n_{i m}}\right)$ (respectively, $X_{m}^{i}{ }^{+}=G O\left(k_{i m}+1, V_{n_{i m}}\right)$ or $X_{m}^{i+}=G S\left(k_{i m}+1, V_{n_{i m}}\right)$ ). Consider the flag variety $\Sigma_{i m}:=\left\{\left(V_{k_{i m}}, V_{k_{i m}+1}\right) \in X_{m}^{i} \times X_{m}^{i+} \mid V_{k_{i m}} \subset V_{k_{i m}+1}\right\}$ with natural projections $X_{m}^{i}{ }^{+} \leftarrow \Sigma_{i m} \rightarrow X_{m}^{i}$. There are induced projections

$$
X_{m}^{i+} \times \widehat{X}_{m}^{i} \stackrel{p_{i m}}{\leftarrow} \Sigma_{i m} \times \widehat{X}_{m}^{i} \stackrel{q_{i m}}{\rightarrow} X_{m}^{i} \times \widehat{X}_{m}^{i} \simeq \widehat{X}_{m}, \quad i \in \Xi
$$

and we put

$$
\pi_{i m}:=\left.p_{i m}\right|_{q_{i m}^{-1}\left(X_{m}\right)}: q_{i m}^{-1}\left(X_{m}\right) \rightarrow X_{m}^{i+} \times \widehat{X}_{m}^{i}, \quad i \in \Xi .
$$

Now let $\left\{c_{m}\right\}_{m \geq 1}$ be a nondecreasing sequence of integers satisfying the conditions

$$
1 \leq c_{m} \leq \min \left\{\frac{k_{i m}-1}{2} \mid i \in \Xi\right\}
$$


where $X_{m}^{i}=G\left(k_{i m}, V_{n_{i m}}\right), G O\left(k_{i m}, V_{n_{i m}}\right), G S\left(k_{i m}, V_{n_{i m}}\right)$. For each $m \geq 1$ consider a linear section $X_{m}$ of $\underset{i \in \Xi}{\times} X_{m}^{i}$

$$
X_{m}:=\left(\underset{i \in \Xi}{\times} X_{m}^{i}\right) \cap \mathbb{P}^{N_{m}-c_{m}-1},
$$

where $\mathbb{P}^{N_{m}-c_{m}-1}=\mathbb{P}\left(U_{m}\right)$ for $U_{m} \in G\left(N_{m}-c_{m}, V_{N_{m}}\right)$. We call $X_{m}$ a linear section of $\underset{i \in \Xi}{\times} X_{m}^{i}$ of codimension $c_{m}$.

Proposition 6.2. For a given $m \geq 1$ such that $k_{i m} \geq 2$ for all $i \in \Xi$, fix an integer $c_{m}$ satisfying (52). Then for a projective subspace $\mathbb{P}^{N_{m}-c_{m}-1}=\mathbb{P}\left(U_{m}\right)$ of general position in $\mathbb{P}^{N_{m}-1}$, $U_{m} \in G\left(N_{m}-c_{m}, V_{N_{m}}\right)$, and any $i \in \Xi$ the following statements hold:

(i) the varieties $X_{m}$ and $q_{i m}^{-1}\left(X_{m}\right)$ are smooth;

(ii) $\operatorname{codim} \underset{i \in \Xi}{\times X_{m}^{i}} X_{m}=\operatorname{codim}_{\Sigma_{i m} \times \widehat{X}_{m}^{i}} q_{i m}^{-1}\left(X_{m}\right)=c_{m}, \pi_{i m *} \mathcal{O}_{q_{i m}^{-1}\left(X_{m}\right)}=\mathcal{O}_{X_{m}^{i}{ }^{+} \times \widehat{X}_{m}^{i}}$, and, for $a$ point $x=\left(x_{1}, x_{2}\right) \in X_{m}^{i} \times \widehat{X}_{m}^{i}$ in general position, the projective subspace $\mathbb{P}^{k_{i m}}=q_{i m} p_{i m}^{-1}(x)$ of $X_{m}^{i} \times\left\{x_{2}\right\}$ satisfies the condition

$$
\operatorname{codim}_{\mathbb{P}^{k_{i m}}}\left(\mathbb{P}^{k_{i m}} \cap \mathbb{P}^{N_{m}-c_{m}-1}\right)=c_{m},
$$

so that $Z_{m}^{i}\left(U_{m}\right):=\left\{x \in X_{m}^{i+} \times \widehat{X}_{m}^{i} \mid \operatorname{dim} \pi_{i m}^{-1}(x)>k_{i m}-c_{m}\right\}$ is a proper closed subset of $X_{m}^{i+} \times \widehat{X}_{m}^{i}$ 3 ;

(iii) let $B_{m}^{i}\left(U_{m}\right):=\pi_{i m}^{-1}\left(Z_{m}^{i}\left(U_{m}\right)\right)$; then $\operatorname{codim}_{q_{i m}^{-1}\left(X_{m}\right)} B_{m}^{i}\left(U_{m}\right) \geq 2, \operatorname{codim}_{X_{m}^{i}+\times \widehat{X}_{m}^{i}} Z_{m}^{i}\left(U_{m}\right) \geq$

(iv) the projection $\pi_{i m}: q_{i m}^{-1}\left(X_{m}\right) \backslash B_{m}^{i}\left(U_{m}\right) \rightarrow X_{m}^{i}{ }^{+} \times \widehat{X}_{m}^{i} \backslash Z_{m}^{i}\left(U_{m}\right)$ is a projective $\mathbb{P}^{k_{i m}-c_{m}}$ bundle.

Proof. Similar to the proof of Proposition 5.1 .

Corollary 6.3. Under the assumptions of Proposition 6.2 , let $i \in \Xi$ and let $\mathcal{E}$ be a vector bundle on $q_{i m}^{-1}\left(X_{m}\right)$ trivial along the fibres of the morphism $\pi_{i m}: q_{i m}^{-1}\left(X_{m}\right) \rightarrow X_{m}^{i+} \times \widehat{X}_{m}^{i}$. Then the sheaf $\pi_{i m *} \mathcal{E}$ is locally free and $e v: \pi_{i m}^{*} \pi_{i m *} \mathcal{E} \stackrel{\simeq}{\rightarrow} \mathcal{E}$ is an isomorphism.

Proof. Proposition 6.2 implies that the data $X=X_{m}^{i+} \times \widehat{X}_{m}^{i}, Y=q_{i m}^{-1}\left(X_{m}\right), B=B_{m}^{i}\left(U_{m}\right)$, $Z=Z_{m}^{i}\left(U_{m}\right), E=\mathcal{E}$ satisfy the conditions of Proposition 7.2 from the appendix. Therefore this latter proposition yields the corollary.

Below we will need the following lemma, the proof of which is similar to that of Lemma 5.3.

Lemma 6.4. For $i=1,2$, let $X_{i}=G\left(k_{i}, V_{i}\right), G O\left(k_{i}, V_{i}\right), G S\left(k_{i}, V_{i}\right)$ and let $X$ be a linear section of codimension $c$ of $X_{1} \times X_{2}$, where $1 \leq c \leq \min \left\{\frac{k_{1}+1}{2}, \frac{k_{2}+1}{2}\right\}$. Then for any projective line $\mathbb{P}_{i}^{1} \subset X_{i}$ there exists a projective line $\mathbb{P}^{1}$ such that pr $\left.\right|_{\mathbb{P}^{1}}$ is an isomorphism of $\mathbb{P}^{1}$ and $\mathbb{P}_{i}^{1}$. Here $\mathrm{pr}_{i}$ stands for the natural projection $X_{1} \times X_{2} \rightarrow X_{i}$.

Consider an ind-variety $\mathbf{X}=\lim _{\rightarrow} X_{m}$ such that, for each $m \geq 1, X_{m}$ is a smooth linear of codimension $c_{m}$ of $\underset{i \in \Xi}{\times} X_{i m}$ where $\vec{c}_{m}$ satisfies (152), and the embeddings $\varphi_{m}: X_{m} \hookrightarrow X_{m+1}$ are induced by the corresponding embeddings $\underset{i \in \Xi}{\times} X_{m}^{i} \hookrightarrow \underset{i \in \Xi}{\times} X_{m+1}^{i}$. In what follows we call $\mathbf{X}$ a linear section of $\underset{i \in \Xi}{\times \mathbf{X}^{i}}$ of small codimension. The existence of linear sections $\mathbf{X}$ of $\underset{i \in \Xi}{\times \mathbf{X}^{i}}$ of small codimension follows immediately from the Bertini Theorem.

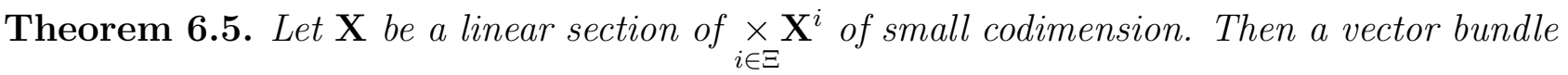
on $\mathbf{X}$ is a direct sum of line bundles.

Proof. We give a proof for the case when all $\mathbf{X}^{i}$ are isomorphic to $\mathrm{GO}(\infty, \infty)$. The case when some $\mathbf{X}^{i}$ are isomorphic to $\mathbf{G}(\infty)$ or $\mathbf{G S}(\infty, \infty)$ is treated similarly. 
First we construct families $\mathbf{B}_{i}, i \in \Xi$, on $\mathbf{X}$. For each $m \geq 1$ and each $i, 1 \leq i \leq l$, consider the natural projection $p_{i m}: X_{m} \rightarrow \hat{X}_{m}^{i}$, and for an arbitrary point $y \in \hat{X}_{m}^{i}$ set $\bar{X}_{m}^{i}(y):=p_{i m}^{-1}(y)$. By definition, $X_{m}^{i}(y)$ is a linear section of the grassmannian $G O\left(k_{i m}, V_{n_{i m}}\right)$. Let $B_{i}(m)(y)$ be the family of projective lines in $G O\left(k_{i m}, V_{n_{i m}}\right)$ lying on $X_{m}^{i}(y)$, and set $B_{i}(m):=\bigcup_{y \in \hat{X}_{m}^{i}} B_{i}(m)(y)$. Then $\mathbf{B}_{i}:=\lim B_{i}(m), i \in \Xi$. Furthermore, the ind-varieties $\boldsymbol{\Pi}_{i}, i \in \Xi$, parametrizing certain families of ind-projective spaces $\mathbb{P}^{\infty}$ in $\mathbf{B}_{i}$, are defined in the same way as the ind-variety $\boldsymbol{\Pi}$ in subsection 5.2 - see (47).

Next, recall the collection of line bundles (49) on $\times \mathbf{X}^{i}$. In our case $\Theta_{\mathbf{X} \xi}$ consists of a single point for each $i$, hence we can write simply $\mathbf{L}=\left\{\mathbf{L}_{i}\right\}_{i \in \Xi}$. We now define a family of line bundles $\mathbf{L}_{\mathbf{X}}$ by putting $\mathbf{L}_{\mathbf{X}}:=\left\{\left.\mathbf{L}_{i}\right|_{\mathbf{X}}\right\}_{i \in \Xi}$. Then by the Lefschetz Theorem $\mathbf{L}_{\mathbf{X}}$ freely generates PicX; in addition, the relation (2) is clearly satisfied. To see that $\mathbf{X}$ satisfies the property $\mathrm{L}$, it remains to notice that $H^{1}\left(X_{m},\left.\underset{i \in \Xi}{\otimes} \mathbf{L}_{i}^{\otimes a_{i}}\right|_{X_{m}}\right)=0$ for all $a_{i}$. Indeed, the vanishing of $H^{1}\left(\underset{i \in \Xi}{\times} X_{m}^{i},\left.\underset{i \in \Xi}{\otimes} \mathbf{L}_{i}^{\otimes a_{i}}\right|_{i \in \Xi} X_{m}^{i}\right)$ follows from Kunneth's and Bott's formulas. Since $\left.\underset{i \in \Xi}{\otimes} \mathbf{L}_{i}^{\otimes a_{i}}\right|_{X_{m}}$ admits a Koszul resolution similar to (34), this is sufficient to conclude that $H^{1}\left(X_{m},\left.\underset{i \in \Xi}{\otimes} \mathbf{L}_{i}^{\otimes a_{i}}\right|_{X_{m}}\right)=0$.

Using Proposition 6.2 and repeating the argument from subsection 5.2, it is easy to check that $\mathbf{X}$ satisfies the property A. Let us show that $\mathbf{X}$ satisfies the property T. The case $|\Xi|=1$ was treated in Proposition 4.4(ii). It is enough to give the proof for the case $|\Xi|=2$; the proof for $|\Xi| \geq 3$ goes along the same lines. We thus assume that $X_{m}$ is a linear section of $X_{m}^{1} \times X_{m}^{2}$. According to (50) and (51) we have a commutative diagram

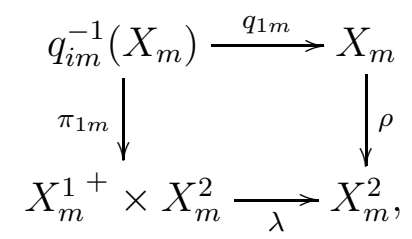

where $l=2, i=1$, so that $\hat{X}_{m}^{1}=X_{m}^{2}$, and $\lambda$ and $\rho$ are the natural projections.

Let $\lim _{\leftarrow} E_{m}$ be a $\mathbf{B}_{i}$-trivial vector bundle on $\mathbf{X}=\lim _{\rightarrow} X_{m}$ for $i=1,2$. This means that each vector bundle $E_{m}$ is a $B_{i}(m)$-trivial bundle on $X_{m}$, i.e. $\left.E_{m}\right|_{\mathbb{P}^{1}}$ is trivial for any $\mathbb{P}^{1} \in B_{i}(m)$, $i=1,2$. Consider the vector bundle

$$
\tilde{E}_{m}:=q_{1 m}^{*} E_{m} .
$$

Since $E_{m}$ is $B_{1}(m)$-trivial, i.e. linearly trivial on the fibres of $\rho, E_{m}$ is trivial by Proposition 5.4. It follows that $\tilde{E}_{m}$ is trivial along the fibres of $\pi_{1 m}$. Therefore, by Corollary 6.3 there is an isomorphism

$$
e v: \pi_{1 m}^{*} \pi_{1 m *} \tilde{E}_{m} \stackrel{\simeq}{\rightarrow} \tilde{E}_{m} .
$$

Moreover, as in the proof of Proposition 5.4, we obtain that the bundle $\left.\pi_{1 m *} \tilde{E}_{m}\right|_{\lambda^{-1}(y)}$ is trivial for any $y \in X_{m}^{2}$.

Thus Proposition 7.1 below and diagram (53) imply that $E_{m}^{\lambda}:=\lambda_{*} \pi_{1 m *} \tilde{E}_{m}$ is a vector bundle on $X_{m}^{2}$ such that $\tilde{E}_{m} \simeq \pi_{1 m}^{*} \lambda^{*} E_{m}^{\lambda} \simeq q_{1 m}^{*} \rho^{*} E_{m}^{\lambda}$. Applying again Proposition 7.1 we obtain

$$
E_{m} \simeq q_{1 m *} \tilde{E}_{m} \simeq q_{1 m *} q_{1 m}^{*} \rho^{*} E_{m}^{\lambda} \simeq \rho^{*} E_{m}^{\lambda} .
$$

Since $E_{m}$ is $B_{2}(m)$-trivial, it follows from (54) and Lemma 6.4 that $E_{m}^{\lambda}$ is a linearly trivial bundle on $X_{m}^{2}$. Hence, $E_{m}^{\lambda}$ is trivial by Proposition 7.4 below, and $E_{m}$ is trivial as well. In this we showed that $\mathbf{X}$ satisfies the property $\mathrm{T}$. 


\subsection{Ind-varieties of generalized flags.}

We first recall some basic definitions concerning generalized flags in a vector space, see [DiP, sections 3-5]. Let $V$ be a countable-dimensional vector space. A set $\mathcal{C}$ of pairwise distinct subspaces of $V$ is called a chain if it is linearly ordered by inclusion. A chain $\mathcal{F}$ of subspaces of $V$ is a generalized flag in $V$ if it satisfies the following conditions:

(i) each $F \in \mathcal{F}$ has an immediate successor or an immediate predecessor, i.e. $\mathcal{F}=\mathcal{F}^{\prime} \cup \mathcal{F}^{\prime \prime}$, where $\mathcal{F}^{\prime} \subset \mathcal{F}$ (respectively, $\mathcal{F}^{\prime \prime} \subset \mathcal{F}$ ) is the set of subspaces in $\mathcal{F}$ having an immediate successor (respectively, predecessor);

(ii) $V \backslash\{0\}=\sqcup_{F^{\prime} \in \mathcal{F}^{\prime}}\left(F^{\prime \prime} \backslash F^{\prime}\right)$, where $F^{\prime \prime} \in \mathcal{F}^{\prime \prime}$ is the immediate successor of $F^{\prime} \in \mathcal{F}^{\prime}$.

We define a flag in $V$ to be a generalized flag in $V$ which is isomorphic as an ordered set to a subset of $\mathbb{Z}$. A flag can be equivalently defined as a chain of subspaces of $V$ such that $\cap_{F \in \mathcal{F}} F=0, \cup_{F \in \mathcal{F}} F=V$ and there exists a strictly monotonic map of ordered sets $\varphi: \mathcal{F} \rightarrow \mathbb{Z}$.

If $\mathcal{F}$ is a generalized flag in $V$ and $\left\{e_{\alpha}\right\}_{\alpha \in A}$ is a basis of $V$ ( $A$ being a countable set), we say that $\mathcal{F}$ and $\left\{e_{\alpha}\right\}_{\alpha \in A}$ are compatible if there exists a strict partial order $\prec$ on $A$ such that, for any $F^{\prime} \in \mathcal{F}^{\prime}, F^{\prime}=\operatorname{Span}\left\{e_{\beta} \mid \beta \prec \alpha\right\}$ for a certain $\alpha \in A$, and $F^{\prime \prime}=F^{\prime} \oplus \operatorname{Span}\left\{e_{\gamma} \mid \gamma\right.$ is not $\prec$-comparable to $\alpha$ \}.

For the rest of this section we fix a basis $E=\left\{e_{n}\right\}$ of $V$. We call a generalized flag $\mathcal{F}$ weakly compatible with $E$ if $\mathcal{F}$ is compatible with a basis $L$ of $V$ such that $E \backslash(E \cap L)$ is a finite set. Furthermore, we define two generalized flags $\mathcal{F}$ and $\mathcal{G}$ in $V$ to be E-commensurable if both $\mathcal{F}$ and $\mathcal{G}$ are weakly compatible with $E$ and there exists an inclusion preserving bijection $\varphi: \mathcal{F} \rightarrow \mathcal{G}$ and a finite-dimensional subspace $U$ in $V$ such that, for every $F \in \mathcal{F}$,

(i) $F \subset \varphi(F)+U, \varphi(F) \subset F+U$, and

(ii) $\operatorname{dim}(F \cap U)=\operatorname{dim}(\varphi(F) \cap U)$.

Let $\mathcal{F l}(\mathcal{F}, E)$ be the set of all generalized flags in $V$ that are $E$-commensurable with $\mathcal{F}$. Following [DiP, Prop. 5.2]) we endow $\mathcal{F} l(\mathcal{F}, E)$ with a structure of an ind-variety in the following way. For any $m \geq 1$ denote $E_{m}:=\left\{e_{\alpha}\right\}_{\alpha \leq m}, V_{m}:=\operatorname{Span}\left(E_{m}\right), E_{m}^{c}:=\left\{e_{\alpha}\right\}_{\alpha>m}$, $V_{m}^{c}:=\operatorname{Span}\left(E_{m}^{c}\right)$. Next, for any $\mathcal{G} \in \mathcal{F} l(\mathcal{F}, E)$ choose a positive integer $m_{\mathcal{G}}$ such that $\mathcal{F}$ and $\mathcal{G}$ are compatible with bases containing $E_{m_{\mathcal{G}}}^{c}$, and $V_{m_{\mathcal{G}}}$ contains a finite-dimensional subspace $U$ which together with the corresponding inclusion preserving bijection $\varphi_{\mathcal{G}}: \mathcal{F} \stackrel{\sim}{\rightarrow} \mathcal{G}$ makes $\mathcal{F}$ and $\mathcal{G} E$-commensurable. We can pick $n_{\mathcal{F}}$ so that $n_{\mathcal{F}} \leq m_{\mathcal{G}}$ for every $\mathcal{G} \in \mathcal{F} l(\mathcal{F}, E)$. Set

$$
\mathcal{G}_{m}:=\left\{G \cap V_{m} \mid G \in \mathcal{G}\right\} \quad n \geq m_{\mathcal{G}} .
$$

The type of the flag $\mathcal{F}_{n}$ yields a sequence of integers

$$
0<d_{1}^{m}<\ldots<d_{s_{m-1}}^{m}<d_{s_{m}}^{m}=m,
$$

and let $\mathcal{F} l\left(d_{m}, V_{m}\right)$ be the usual flag variety of type $d_{m}=\left(d_{1 m}, \ldots, d_{m, s_{m-1}}\right)$ in $V_{m}$. Notice that $s_{m+1}=s_{m}$ or $s_{m+1}=s_{m}+1$. Furthermore, in both cases an integer $j_{m}$ is determined as follows: in the former case $d_{m+1, i}=d_{m, i}$ for $0 \leq i<j_{m}$ and $d_{m+1, i}=d_{m, i}+1$ for $j_{m} \leq i<s_{m}$, and in the latter case $d_{m+1, i}=d_{m, i}$ for $0 \leq i<j_{m}$ and $d_{m+1, i}=d_{m, i-1}+1$ for $j_{m} \leq i<s_{m}$.

Now we define a map $\iota_{m}: \mathcal{F} l\left(d_{m}, V_{m}\right) \rightarrow \mathcal{F} l\left(d_{m+1}, V_{m+1}\right)$ for every $m \geq m_{\mathcal{F}}$. Given a flag $\mathcal{G}_{m}=\left\{0=G_{0}^{m} \subset G_{1}^{m} \subset \ldots \subset G_{s_{m}}^{m}=V_{m}\right\} \in \mathcal{F} l\left(d_{m}, V_{m}\right)$, put $\iota_{m}\left(\mathcal{G}_{m}\right)=\mathcal{G}_{m+1}:=\left\{0=G_{0}^{m+1} \subset\right.$ $\left.G_{1}^{m+1} \subset \ldots \subset G_{s_{m+1}}^{m+1} V_{m+1}\right\}$, where

$$
G_{i}^{m+1}= \begin{cases}G_{i}^{m} & \text { if } 0 \leq i<j_{m} \\ G_{i}^{m} \oplus k e_{m+1} & \text { if } j_{m} \leq i \leq s_{m+1} \text { and } s_{m+1}=s_{m} \\ G_{i-1}^{m} \oplus k e_{m+1} & \text { if } j_{m} \leq i \leq s_{m+1} \text { and } s_{m+1}=s_{m}+1\end{cases}
$$


The maps $\iota_{m}$ are closed embeddings of algebraic varieties, and hence $\lim \mathcal{F} l\left(d_{m}, V_{m}\right)$ is an indvariety. A bijection between $\mathcal{F} l(\mathcal{F}, E)$ and $\lim _{\rightarrow} \mathcal{F} l\left(d_{m}, V_{m} m m\right)$ is given by

$$
\tau: \mathcal{F} l(\mathcal{F}, E) \stackrel{\sim}{\rightarrow} \lim _{\rightarrow} \mathcal{F} l\left(d_{m}, V_{m}\right), \quad \mathcal{G} \mapsto \lim _{\rightarrow} \mathcal{G}_{m}
$$

- see [DiP, Prop. 5.2].

Assume now that $\mathcal{F}$ is a flag of subspaces in $V$. Then $\mathcal{F}=\left\{\ldots \subset F_{i} \subset F_{i+1} \subset \ldots\right\}$, where $\Theta$ is one of the four linearly ordered sets $\{1, \ldots, n\}, \mathbb{Z}, \mathbb{Z}_{>0}, \mathbb{Z}_{<0}$. Assume in addition that

$$
\operatorname{dim}\left(F^{\prime \prime} / F^{\prime}\right)=\infty
$$

for all $i \in \Theta$ for which $i+1 \in \Theta$. Denote by $\hat{\mathcal{F}}(i)$ the flag $\hat{\mathcal{F}} \backslash\left\{F_{i}\right\}=\left\{\ldots \subset F_{i-1} \subset F_{i+1} \subset \ldots\right\}$. There is natural projection $\pi_{i}: \mathcal{F} l(\mathcal{F}, E) \rightarrow \mathcal{F l}(\hat{\mathcal{F}}(i), E)$. Let $\hat{\mathcal{G}}=\left\{\ldots \subset G_{i-1} \subset G_{i+1} \subset\right.$ $\ldots\} \in \mathcal{F} l(\hat{\mathcal{F}}(i), E)$ and $\mathcal{G}=\left\{\ldots \subset G_{i-1} \subset G_{i} \subset G_{i+1} \subset \ldots\right\} \in \pi_{i}^{-1}(\hat{\mathcal{G}})$. Then the fibre $\pi_{i}^{-1}(\hat{\mathcal{G}})$ equals $\mathcal{F l}\left(G_{i} / G_{i-1}, E(i)\right)$ where $E(i)=\left(E \cap G_{i+1}\right) \backslash\left(E \cap G_{i-1}\right)$. Note that the ind-variety $\mathcal{F l}\left(G_{i} / G_{i-1}, E(i)\right)$ is isomorphic to the ind-grassmannian $\mathbf{G}(\infty)$.

Moreover, there is a well-defined line bundle $\mathbf{L}_{i}:=\mathcal{O}_{\pi_{i}}(1):=\lim \mathcal{O}_{\pi_{i m}}(1)$ on $\mathcal{F} l(\mathcal{F}, E)$, where $\pi_{i m}: \mathcal{F l}\left(d_{m}, V_{m}\right) \rightarrow \mathcal{F} l\left(\hat{d}_{m}(i), V_{m}\right)$ is the natural projection and $\hat{d}_{m}(i)$ is defined in the same way as $d_{m}$ using the flag $\hat{\mathcal{F}}(i)$ instead of $\mathcal{F}$. The fact that the line bundles $\mathcal{O}_{\pi_{i m}}(1)$ yield a well-defined bundle $\mathcal{O}_{\pi_{i}}(1)$ is established by a straightforward checking using the explicit form of the embeddings $\iota_{m}$.

By $\mathbf{B}_{i}(\mathcal{G})$ we denote the ind-variety of projective lines on $\mathcal{F l}(\mathcal{F}, E)$ passing through a point $\mathcal{G} \in \mathcal{F} l(\mathcal{F}, E)$ and lying in the fibre of $\pi_{i}$ which contains $\mathcal{G}$. Finally, we define the ind-variety $\boldsymbol{\Pi}_{i}(\mathcal{G})$ as the ind-variety $\boldsymbol{\Pi}(\mathcal{G})$ for the ind-grassmannian $\mathcal{F} l\left(G_{i} / G_{i-1}, E(i)\right) \simeq \mathbf{G}(\infty)$ as defined in subsection 4.3 .

It is easy to check that $\mathcal{F} l(\mathcal{F}, E)$ satisfies the properties $\mathrm{L}, \mathrm{A}$ and $\mathrm{T}$ with respect to the data $\Theta_{\mathcal{F} l(\mathcal{F}, E)}:=\Theta, \mathbf{L}_{i}, \mathbf{B}_{i}:=\underset{\mathcal{G} \in \mathcal{F} l(\mathcal{F}, E)}{\cup} \mathbf{B}_{i}(\mathcal{G}), \mathbf{\Pi}_{i}:=\underset{\mathcal{G} \in \mathcal{F} l(\mathcal{F}, E)}{\cup} \boldsymbol{\Pi}_{i}(\mathcal{G})$. As a result, Theorem 2.1 implies the following theorem.

Theorem 6.6. Let $V$ be a countable-dimensional vector space with basis $E$. Let $\mathcal{F}$ be a flag in $V$ satisfying (55) weakly compatible with $E$. Then any vector bundle on $\mathcal{F l}(\mathcal{F}, E)$ is isomorphic to a direct sum of line bundles.

It is an interesting question whether the BVTS Theorem holds on any ind-variety of generalized flags $\mathcal{F} l(\mathcal{F}, E)$ under the assumption that the generalized flag $\mathcal{F}$ satisfies (55) for all $F^{\prime} \in \mathcal{F}^{\prime}$ and their respective successors $F^{\prime \prime}$.

\section{Appendix}

In this appendix we collect some general facts about coherent sheaves on projective varieties and their behaviour under flat projective morphisms, which are used throughout the paper.

Proposition 7.1. Let $p: Y \rightarrow X$ be a smooth flat projective morphism of projective varieties with irreducible fibres.

1) If $E$ is a vector bundle on $Y$, trivial on the fibres of $p$, then the evaluation morphism ev $: p^{*} p_{*} E \rightarrow E$ is an isomorphism.

2) If $F$ be a vector bundle on $X$, then the canonical morphism $F \stackrel{\sim}{\rightarrow} p_{*} p^{*} F$ is an isomorphism. 
Proof. 1) This follows easily from the Base-change Theorem [H, Ch. III, Cor. 12.9].

2) Consider the Stein factorization $f: Y \stackrel{f^{\prime}}{\rightarrow} X^{\prime} \stackrel{g}{\rightarrow} X$ of $f$, where $X^{\prime}=\operatorname{Spec}\left(f_{*} \mathcal{O}_{Y}\right)$ and $f_{*}^{\prime} \mathcal{O}_{Y}=\mathcal{O}_{X^{\prime}}$ (see [H1, Ch. III, Cor.12.9]). Since $f_{*} \mathcal{O}_{Y}$ is an invertible sheaf by 1), it follows that $g$ is an isomorphism. Therefore $f_{*} \mathcal{O}_{Y}=\mathcal{O}_{X}$. This, together with the projection formula [H1, Ch. III, Exc. 8.3], gives the desired assertion.

Proposition 7.2. Let $\pi: Y \rightarrow X$ be a surjective morphism of smooth irreducible projective varieties such that:

(i) the fibres of $\pi$ are projective spaces;

(ii) the variety $Z:=\left\{x \in X \mid \operatorname{dim} \pi^{-1}(x)>\operatorname{dim} Y-\operatorname{dim} X\right\}$ has codimension at least 3 in $X$, and the variety $B:=\pi^{-1}(Z)$ has codimension at least 2 in $Y$;

(iii) there exists a vector bundle $F$ on $X \backslash Z$ such that $\pi: Y \backslash B \simeq \mathbb{P}(F) \rightarrow X \backslash Z$ is the structure map of the projectivized vector bundle $F$.

Next, let $E$ be a vector bundle on $Y$, trivial along the fibres of $\pi$. Then the $\mathcal{O}_{X}$-sheaf $\pi_{*} E$ is locally free and the evaluation morphism ev $: \pi^{*} \pi_{*} E \rightarrow E$ is an isomorphism.

Proof. We first show that $e v: \pi^{*} \pi_{*} E \rightarrow E$ is an isomorphism. For this, consider an arbitrary open subvariety $U \subset X$ and its closed subvariety $A \subset U$ such that

$$
\operatorname{codim}_{U} A \geq 2 \text {. }
$$

Since $X$ is smooth and $Z$ has codimension $\geq 3$ in $X$, it follows that $\operatorname{codim}_{U}(Z \cap A) \geq 3$ and

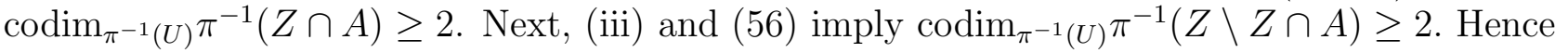

$$
\operatorname{codim}_{\pi^{-1}(U)} \pi^{-1}(A) \geq 2 .
$$

Let $s \in H^{0}\left(U \backslash A,\left.\pi_{*} E\right|_{U \backslash A}\right)$ and let $\tilde{s}:=\varphi(s)$, where $\varphi: H^{0}\left(U \backslash A,\left.\pi_{*} E\right|_{U \backslash A}\right) \stackrel{\widetilde{\sim}}{\rightarrow} H^{0}\left(\pi^{-1}(U \backslash\right.$ $\left.A),\left.E\right|_{\pi^{-1}(U \backslash A)}\right)$ is the canonical isomorphism. Since $E$ is a locally free sheaf on a smooth variety $Y, E$ is normal by [H2, Prop. 1.6(ii)], i.e. (57) implies that $\tilde{s}$ extends uniquely to a section $\tilde{s}^{\prime} \in H^{0}\left(\pi^{-1}(U),\left.E\right|_{\pi^{-1}(U)}\right)$. Then $s$ extends to the section $s^{\prime}:=\psi\left(\tilde{s}^{\prime}\right) \in H^{0}\left(U,\left.\pi_{*} E\right|_{U}\right)$, where $\psi: H^{0}\left(\pi^{-1}(U),\left.E\right|_{\pi^{-1}(U)}\right) \stackrel{\sim}{\rightarrow} H^{0}\left(U,\left.\pi_{*} E\right|_{U}\right)$ is the canonical isomorphism. In view of (56) this means that the sheaf $\pi_{*} E$ is normal.

Note that $\pi_{*} E$ is torsion-free. Indeed, if the torsion subsheaf Tor $s\left(\pi_{*} E\right)$ were nonzero, then since $E$ is locally free, by (iii) any section $0 \neq s \in H^{0}\left(Y, \operatorname{Tor} s\left(\pi_{*} E\right)\right)$ would be supported in $Z$. Then the section $0 \neq \tilde{s}:=\psi^{-1}(s)$ would be supported in $B$, i.e. Tors $(E) \neq 0$, This contradicts the assumptions that $E$ is locally free and $Y$ is smooth and irreducible.

Hence, $\pi_{*} E$ is reflexive by [H2, Prop. 1.6]. Set

$$
\tilde{E}:=\pi^{*} \pi_{*} E / \operatorname{Tors}\left(\pi^{*} \pi_{*} E\right) .
$$

Proposition 7.1, together with (iii), implies the existence of an isomorphism

$$
\alpha:\left.\left.\tilde{E}\right|_{Y \backslash B} \stackrel{\simeq}{\rightarrow} E\right|_{Y \backslash B} .
$$

Now by [H2, Prop. 1.1] the sheaf $\pi_{*} E$ can, locally on $X$, be included in an exact sequence

$$
0 \rightarrow \pi_{*} E \rightarrow L_{1} \rightarrow L_{2}
$$

with locally free sheaves $L_{1}$ and $L_{2}$. Applying to (59) the functor $\pi^{*}$ we obtain the sequence

$$
0 \rightarrow \tilde{E} \rightarrow \pi^{*} L_{1} \rightarrow \pi^{*} L_{2}
$$

which is exact when restricted onto $Y \backslash B$. Hence, this sequence is exact as $\tilde{E}$ is torsion free and the sheaves $\pi^{*} L_{1}$ and $\pi^{*} L_{2}$ are locally free. By [H2, Prop. 1.1] this implies that $\tilde{E}$ is reflexive. Therefore, denoting by $i$ the inclusion $Y \backslash B \hookrightarrow Y$ and using the isomorphism (58) and [H2, Prop. 1.6(iii)] we obtain an isomorphism

$$
\pi^{*} \pi_{*} E=\tilde{E} \cong i_{*}\left(\left.\tilde{E}\right|_{Y \backslash B}\right) \stackrel{i_{*} \alpha}{\longrightarrow} i_{*}\left(\left.E\right|_{Y \backslash B}\right) \cong E .
$$


This isomorphism is nothing but the evaluation morphism $e v$.

It remains to show that $\pi_{*} E$ is locally free. The isomorphism $e v$ implies that $\pi^{*} \pi_{*} E$ is locally free. Therefore, for any $y \in Y, r:=\operatorname{dim}_{\mathbb{C}(y)}\left(\pi^{*} \pi_{*} E \otimes \mathbb{C}(y)\right)$ does not depend on $y$, and consequently, $\operatorname{dim}_{\mathbb{C}(x)}\left(\pi_{*} E \otimes \mathbb{C}(x)\right)=r$. According to [M, $\S 5$, Lemma 1], since $X$ is smooth, the fact that $\operatorname{dim}_{\mathbb{C}(x)}\left(\pi_{*} E \otimes \mathbb{C}(x)\right)$ does not depend on $x \in X$ implies that $\pi_{*} E$ is locally free.

Proposition 7.3. Let $Q_{n}$ be a nonsingular $n$-dimensional quadric in $\mathbb{P}^{n+1}$ for $n \geq 2$, and let $E$ be a linearly trivial vector bundle on $Q_{n}$. Then $E$ is trivial.

Proof. We argue by induction on $n$. For $n=2$ the proof is easy and is the same as for a projective space as given in OSS, Ch. I, Thm. 3.2.1]. Thus we may assume that $n \geq 3$. Consider a codimension-2 subspace $\mathbb{P}^{n-1}$ in $\mathbb{P}^{n+1}$ such that $Q_{n-2}:=Q_{n} \cap \mathbb{P}^{n-1}$ is a smooth quadric of dimension $n-2$. If $n \geq 4$ then $\left.E\right|_{l}$ is trivial for any projective line $l \subset Q_{n-2}$, hence $\left.E\right|_{Q_{n-2}}$ is trivial by the induction assumption. For $n=3$ the quadric $Q_{n-2}$ is a smooth conic $C$. By Bertini's Theorem there exists a smooth quadric surface $Q_{2}$ on $Q_{3}$ passing through the conic $C$. Since $\left.E\right|_{Q_{2}}$ is trivial (being linearly trivial), $\left.E\right|_{C}$ is also trivial, i.e. our claim holds for $n=3$.

We will now use the triviality of $\left.E\right|_{Q_{n-2}}$ for $n \geq 4$ to show that $E$ is trivial. Let $\sigma_{Q}: \tilde{Q}_{n} \rightarrow Q_{n}$ be the blow-up of $Q_{n}$ with center at $Q_{n-2}$, and let $D:=\sigma_{Q}^{-1}\left(Q_{n-2}\right)$ be the exceptional divisor. Clearly, $D \simeq Q_{n-2} \times \mathbb{P}^{1}$ and there is a flat surjective morphism $\pi: \tilde{Q}_{n} \rightarrow \mathbb{P}^{1}$ fitting in the commutative diagram

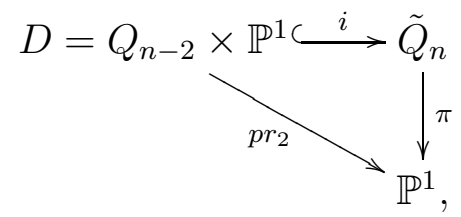

where $i$ is the embedding of the exceptional divisor. By construction, there exist two distinct points $t_{1}, t_{2} \in \mathbb{P}^{1}$ such that the fibre $Q_{n-1}(t)=\pi^{-1}(t)$ is a smooth quadric for $t \in U:=$ $\mathbb{P}^{1} \backslash\left\{t_{1} \cup t_{2}\right\}$, and $Q_{n-1}\left(t_{j}\right):=\pi^{-1}\left(t_{j}\right)$ for $j=1,2$ are quadratic cones whose vertices are points.

Consider the vector bundle $\tilde{E}:=\sigma_{Q}^{*} E$ on $\tilde{Q}_{n}$. By construction, $\tilde{E}$ is trivial on any projective line $l \subset Q_{n-1}(t), t \in U$. Hence, by the induction assumption, $\left.E\right|_{Q_{n-1}(t)}, t \in U$, is trivial. Consequently,

(61)

$H^{i}\left(Q_{n-1}(t),\left.\tilde{E}(-D)\right|_{Q_{n-1}(t)}\right)=0, i \geq 0, \quad \operatorname{dim} H^{i}\left(Q_{n-1}(t),\left.\tilde{E}\right|_{Q_{n-1}(t)}\right)=\left\{\begin{array}{ll}r, & \text { if } i=0, \\ 0, & \text { if } i \geq 1,\end{array} \quad t \in U\right.$.

Next, for $j=1,2$, let $\sigma: K_{j} \rightarrow Q_{n-1}\left(t_{j}\right)$ be the blow-up of the cone $Q_{n-1}\left(t_{j}\right)$ with center at the singular point. Let $f_{j}: K_{j} \rightarrow Q_{n-2}$ be the induced $\mathbb{P}^{1}$-bundle, the fibres of which map to projective lines on $Q_{n-1}\left(t_{j}\right)$ under the morphism $\sigma$. Since $\tilde{E}_{t_{j}}:=\left.\tilde{E}\right|_{Q_{n-1}\left(t_{j}\right)}$ is trivial along the projective lines on $Q_{n-1}\left(t_{j}\right)$, it follows that the bundle $\tilde{E}_{K_{j}}:=\sigma^{*} \tilde{E}_{t_{j}}$ is trivial along the fibers of $f_{j}$. Therefore, for an arbitrary point $x \in Q_{n-2}$ we obtain

$$
\begin{gathered}
H^{i}\left(Q_{n-2}, \tilde{E}_{K_{j}} \otimes \mathbb{C}_{x}\right)=H^{i}\left(\mathbb{P}^{1}, r \mathcal{O}_{\mathbb{P}^{1}}\right)=0, \quad i \geq 1, \\
H^{i}\left(Q_{n-2}, \tilde{E}_{K_{j}}\left(-\sigma^{*} D\right) \otimes \mathbb{C}_{x}\right)=H^{i}\left(\mathbb{P}^{1}, r \mathcal{O}_{\mathbb{P}^{1}}(-1)\right)=0, \quad i \geq 0 .
\end{gathered}
$$

This, together with the Base-change Theorem for $f_{j}$, shows that $R^{i} f_{j *} \tilde{E}_{K_{j}}=0, i \geq$ 1, $\quad R^{i} f_{j *}\left(\tilde{E}_{K_{j}}\left(-\sigma^{*} D\right)\right)=0, \quad i \geq 0$. Hence the Leray spectral sequence for the projection $f_{j}$ yields

$$
H^{i}\left(K_{j}, \tilde{E}_{K_{j}}\right)=0, \quad i \geq 1, \quad H^{i}\left(K_{j}, \tilde{E}_{K_{j}}\left(-\sigma^{*} D\right)\right)=0, \quad i \geq 0, \quad j=1,2 .
$$


Next, one uses the embedded in $\mathbb{P}^{n}$ blow-up of the cone $Q_{n-1}\left(t_{j}\right)$ that $\sigma_{*} \mathcal{O}_{K_{j}}=\mathcal{O}_{Q_{n-1}\left(t_{j}\right)}$ and $R^{i} \sigma_{*} \mathcal{O}_{K_{j}}=0, i \geq 1$. Therefore, setting $\tilde{E}_{t_{j}}:=\left.\tilde{E}\right|_{Q_{n-1}\left(t_{j}\right)}$, we have by the projection formula: $\sigma_{*} \tilde{E}_{K_{j}}=\tilde{E}_{t_{j}}, R^{i} \sigma_{*} \tilde{E}_{K_{j}}=0, i \geq 1$, and $\sigma_{*}\left(\tilde{E}_{K_{j}}\left(-\sigma^{*} D\right)\right)=\tilde{E}_{t_{j}}(-D), R^{i} \sigma_{*}\left(\tilde{E}_{K_{j}}\left(-\sigma^{*} D\right)\right)=$ $0, i \geq 1$. Now the Leray spectral sequence applied to $\sigma$ shows in view of (62) that

$$
\begin{array}{cc}
H^{i}\left(Q_{n-1}\left(t_{j}\right), \tilde{E}_{t_{j}}\right)=H^{i}\left(K_{j}, \tilde{E}_{K_{j}}\right)=0, & i \geq 1, \\
H^{i}\left(Q_{n-1}\left(t_{j}\right), \tilde{E}_{t_{j}}(-D)\right)=H^{i}\left(K_{j}, \tilde{E}_{K_{j}}\left(-\sigma^{*} D\right)\right)=0, & i \geq 0, \quad j=1,2 .
\end{array}
$$

The equalities (61) and (63) yield via base change for the flat morphism $\pi$

$$
R^{i} \pi_{*} \tilde{E}=0, i \geq 1, \quad R^{i} \pi_{*}(\tilde{E}(-D))=0, \quad i \geq 0 .
$$

The same argument yields base change isomorphisms

$$
b_{t}: \pi_{*} \tilde{E} \otimes \mathbb{C}_{t} \stackrel{\simeq}{\rightarrow} H^{0}\left(Q_{n-1}(t),\left.\tilde{E}\right|_{Q_{n-1}(t)}\right), \quad t \in \mathbb{P}^{1} .
$$

Consider the divisor $D=Q_{n-2} \times \mathbb{P}^{1}$ on $\tilde{Q}_{n}$ (see diagram (60) $)$ and the projections $Q_{n-2} \stackrel{p r_{1}}{\leftarrow}$ $Q_{n-2} \times \mathbb{P}^{1} \stackrel{p r_{2}}{\rightarrow} \mathbb{P}^{1}$. By definition, $\left.\tilde{E}\right|_{D}=\operatorname{pr}_{1}^{*}\left(\left.\tilde{E}_{m}\right|_{Q_{n-2}}\right)$, hence, since $\left.\tilde{E}\right|_{Q_{n-2}}$ is trivial, the base change for the flat morphism $p r_{2}$ gives the isomorphisms

$$
\begin{aligned}
& b^{\prime}: \operatorname{pr}_{2 *}\left(\left.\tilde{E}\right|_{D}\right) \stackrel{\simeq}{\rightarrow} H^{0}\left(Q_{n-2},\left.E\right|_{Q_{n-2}}\right) \otimes \mathcal{O}_{\mathbb{P}^{1}} \simeq \mathbb{C}^{r} \otimes \mathcal{O}_{\mathbb{P}^{1}}, \\
& b_{t}^{\prime}: \operatorname{pr}_{2 *}\left(\left.\tilde{E}\right|_{D}\right) \otimes \mathbb{C}_{t} \stackrel{\simeq}{\rightarrow} H^{0}\left(Q_{n-2},\left.E\right|_{Q_{n-2}}\right) \simeq \mathbb{C}^{r}, \quad t \in \mathbb{P}^{1} .
\end{aligned}
$$

Now consider the exact triple

$$
\left.0 \rightarrow \tilde{E}(-D) \rightarrow \tilde{E} \rightarrow E\right|_{D} \rightarrow 0
$$

and its restriction onto a fibre $Q_{n-1}(t)$ of the projection $\pi$ over an arbitrary point $t \in \mathbb{P}^{1}$

$$
\left.\left.0 \rightarrow \tilde{E}(-D)\right|_{Q_{n-1}(t)} \rightarrow \tilde{E}\right|_{Q_{n-1}(t)} \rightarrow\left(\left.E\right|_{Q_{n-2}}\right) \otimes \mathbb{C}_{t} \rightarrow 0 .
$$

Applying the functor $R^{i} \pi_{*}$ to (68) and using (64) and (66) we obtain the isomorphism of sheaves

$$
r_{D}: \pi_{*} \tilde{E} \stackrel{\sim}{\rightarrow} \operatorname{pr}_{2 *}\left(\left.\tilde{E}\right|_{D}\right) \simeq \mathbb{C}^{r} \otimes \mathcal{O}_{\mathbb{P}^{1}} .
$$

In particular, $\pi_{*} \tilde{E}$ is a trivial bundle. Respectively, passing to cohomology of the exact sequence (69) and using (61), (63) and (67), we obtain the isomorphisms

$$
r e s_{t}: H^{0}\left(Q_{n-1}(t),\left.\tilde{E}\right|_{Q_{n-1}(t)}\right) \stackrel{\sim}{\rightarrow} H^{0}\left(Q_{n-2},\left.E\right|_{Q_{n-2}}\right), \quad t \in \mathbb{P}^{1} .
$$

By construction the isomorphisms (65), (67), (70) and (71) fit in the commutative diagram

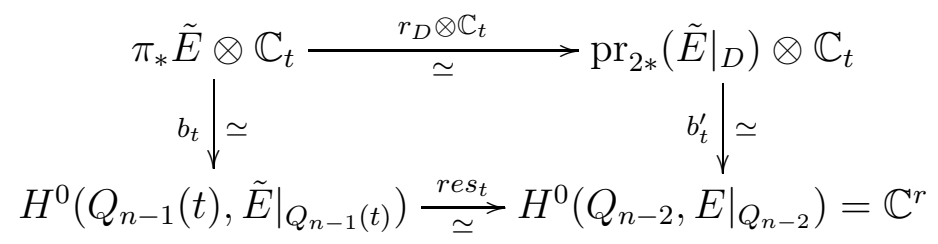

for $t \in \mathbb{P}^{1}$. Next, since $\left.E\right|_{Q_{n-2}}$ is trivial, the evaluation map $\left.H^{0}\left(Q_{n-2},\left.E\right|_{Q_{n-2}}\right) \otimes \mathcal{O}_{Q_{n-2}} \rightarrow E\right|_{Q_{n-2}}$ is an isomorphism, so that its composition $e_{t}$ with the restriction $H^{0}\left(Q_{n-2},\left.E\right|_{Q_{n-2}}\right) \otimes \mathcal{O}_{Q_{n-1}(t)} \rightarrow$ $H^{0}\left(Q_{n-2},\left.E\right|_{Q_{n-2}}\right) \otimes \mathcal{O}_{Q_{n-2}}$ is an epimorphism for any $t \in \mathbb{P}^{1}$ and fits in the commutative diagram

$$
\begin{gathered}
H^{0}\left(Q_{n-1}(t),\left.\tilde{E}\right|_{Q_{n-1}(t)}\right) \otimes \mathcal{O}_{Q_{n-1}(t)} \stackrel{\pi^{*} \text { rest }}{\simeq} H^{0}\left(Q_{n-2},\left.E\right|_{Q_{n-2}}\right) \otimes \mathcal{O}_{Q_{n-1}(t)} \\
e v_{t} \downarrow \\
\left.\left.\tilde{E}\right|_{Q_{n-1}(t)} \longrightarrow E\right|_{Q_{n-2}} .
\end{gathered}
$$


Here we understand $Q_{n-2}$ as lying in $Q_{n-1}(t)$ as a divisor. In particular, through any point of $Q_{n-1}(t) \backslash Q_{n-2}$ there passes a line, say, $l$ interesecting $Q_{n-2}$ at a point, say $y$. Therefore, since $\left.\tilde{E}\right|_{l}$ is trivial, we have a commutative diagram of restriction maps

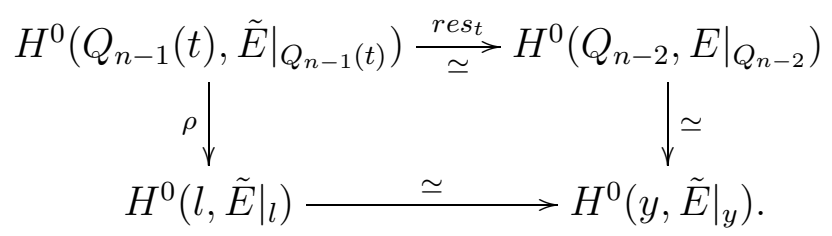

Hence, $\rho$ is an isomorphism, and therefore the evaluation morphism $e v_{t}$ in (173) is an isomorphism of sheaves. Composing it with the isomorphism $\pi^{*} b_{t}:\left.\pi^{*} \pi_{*} \tilde{E}\right|_{Q_{n-1}(t)} \stackrel{\widetilde{ }}{\rightarrow} H^{0}\left(Q_{n-1}(t),\left.\tilde{E}\right|_{Q_{n-1}(t)}\right) \otimes$ $\mathcal{O}_{Q_{n-1}(t)}$ arising from the left vertical isomorphism in (72) we obtain the (evaluation) isomorphism $\left.e v\right|_{Q_{n-1}(t)}:\left.\left.\pi^{*} \pi_{*} \tilde{E}\right|_{Q_{n-1}(t)} \stackrel{\simeq}{\rightarrow} \tilde{E}\right|_{Q_{n-1}(t)}$. Since this is true for any $t \in \mathbb{P}^{1}$, we obtain the isomorphism ev : $\pi^{*} \pi_{*} \tilde{E} \stackrel{\widetilde{\Xi}}{\rightarrow} \tilde{E}$ which together with (70) leads to the triviality of $\tilde{E}$. Since clearly $\sigma_{Q *} \mathcal{O}_{\tilde{Q}}=\mathcal{O}_{Q}$, it follows that $E=\sigma_{Q *} \tilde{E}=\sigma_{Q *}\left(r \mathcal{O}_{\tilde{Q}}\right)=r \mathcal{O}_{Q}$, i. e. we obtain the statement of Proposition.

Proposition 7.4. Let $E$ be a linearly trivial vector bundle on $G O(k, V)$ or $G S(k, V)$. Then $E$ is trivial.

Proof. Consider the case $G O(k, V)$. We give a proof by induction under the assumption that $n:=\frac{\operatorname{dim} V}{2} \in \mathbb{Z}_{>0}$. The case when $\operatorname{dim} V$ is odd can be treated similarly.

For $n=2$ we have $G O(1, V) \simeq \mathbb{P}^{1} \times \mathbb{P}^{1}, G O(2, V) \simeq \mathbb{P}^{1}$, and for these varieties our claim clearly holds. Therefore we assume that $n \geq 3$ and argue by induction on $k$. If $k=1, G O(k, V)$ is a $(2 n-2)$-dimensional quadric in $\mathbb{P}^{2 n-1}$ so our statement holds by Proposition 7.3 . Now let $1 \leq k \leq n-2$, and recall the graph of incidence $\Sigma$ with natural projections

$$
G O(k, V) \stackrel{q}{\leftarrow} \Sigma \stackrel{p}{\rightarrow} G O(k+1, V)
$$

(see subsection 5.1).

Let $E$ be a linearly trivial vector bundle on $G O(k+1, V)$. Then the bundle $p^{*} E$ is linearly trivial on the fibres of $q$. Since these fibres are quadrics, Proposition 7.3 implies that $p^{*} E$ is trivial on the fibres of $q$. Furthermore, Proposition 7.1 yields an isomorphism $q^{*} q_{*} p^{*} E \stackrel{\widetilde{\Im}}{\rightarrow} p^{*} E$. Hence, since $p^{*} E$ is trivial along the fibres of $p$ which are mapped by $q$ isomorphically to projective spaces $\mathbb{P}^{k}$ on $G O(k, V)$, it follows that $q_{*} p^{*} E$ is trivial along these projective subspaces $\mathbb{P}^{k}$ of $G O(k, V)$. Consequently, $q_{*} p^{*} E$ is linearly trivial on $G O(k, V)$. Thus, by the induction assumption, $q_{*} p^{*} E$ is trivial. Hence $p^{*} E$ and $E=p_{*} p^{*} E$ are trivial.

It remains to consider $G O(n, V)$. Here we employ induction on $n$. For $n=3 G O(n, V) \simeq \mathbb{P}^{3}$, hence the statement holds in this case. For $n \geq 4$, consider the graph of incidence $\Pi_{n}:=$ $\left\{\left(V_{1}, V_{n}\right) \in Q_{2 n-2} \times G O(n, V) \mid V_{1} \subset V_{n}\right\}$ with natural projections

$$
Q_{2 n-2} \stackrel{p}{\leftarrow} \Pi_{n} \stackrel{q}{\rightarrow} G O(n, V) .
$$

Let $E$ be a linearly trivial vector bundle on $G O(n, V)$. Then $q^{*} E$ is trivial on lines lying in the fibres of $p$ which are isomorphic to $G O\left(n-1, \mathbb{C}^{2 n-2}\right)$. Hence $q^{*} E$ is trivial along the fibres of $p$ by the induction assumption. Next, Proposition 7.1 yields an isomorphism $p^{*} p_{*} q^{*} E \stackrel{\simeq}{\rightarrow} q^{*} E$. Since $q^{*} E$ is trivial on the fibres of $p$, it follows that $p_{*} q^{*} E$ is trivial on the projective subspaces $\mathbb{P}^{n-1}$ of the quadric $Q_{2 n-2}$. Therefore $p_{*} q^{*} E$ is trivial on the lines in $Q_{2 n-2}$, so it is trivial by Proposition 7.3. Finally, $q^{*} E \simeq p^{*} p_{*} q^{*} E$ and $E=q_{*} q^{*} E$ are trivial as well.

Proceed to the case of $G S(k, V)$. Substituting $G O$ by $G S$ in diagram (74), we obtain a diagram $G S(k, V) \stackrel{q}{\leftarrow} \Sigma^{\prime} \stackrel{p}{\rightarrow} G S(k+1, V)$, where $p$ is a $\mathbb{P}^{k}$-bundle and $q$ is a $\mathbb{P}^{2 n-2 k-1}$-bundle. Respectively, substituting $G O$ by $G S$, and $Q_{2 n-2}$ by $\mathbb{P}\left(V_{n}\right)$ in diagram (175), we obtain a diagram $\mathbb{P}\left(V_{n}\right) \stackrel{p}{\leftarrow} \Pi_{n}^{\prime} \stackrel{q}{\rightarrow} G S_{n}$. This enables us to carry out an argument very similar to the one for $G O(k, V)$. 


\section{REFERENCES}

[BV] W. Barth, A. Van de Ven. On the geometry in codimension 2 in Grassmann manifolds. In: Lecture Notes in Math. 412, Springer-Verlag 1974, pp. 1-35.

[DP] J. Donin, I. Penkov. Finite rank vector bundles on inductive limits of grassmannians. IMRN No. 34 (2003), 1871-1887.

[DiP] I. Dimitrov, I. Penkov. Ind-varieties of generalized flags as homogeneous spaces for classical indgroups. IMRN No. 55 (2004), 2935-2953.

[DPW] I. Dimitrov, I. Penkov, J. A. Wolf. A Bott-Borel-Weil theory for direct limits of algebraic groups. Amer. J. Math. 124 (2002), 955-998.

[F] W. Fulton. Intersection Theory, Second Ed. Springer-Verlag, New York, 1998.

[H1] R. Hartshorne. Algebraic Geometry. Springer-Verlag, New York, 1977.

[H2] R. Hartshorne. Stable reflexive sheaves. Math. Ann. 254 (1980), 121-176.

[H3] R. Hartshorne. On the de Rham cohomology of algebraic varieties. Inst. Hautes Études Sci. Publ. Math. 45 (1976), 5-99.

[Ha] J. Harris. Algebraic Geometry: a first course. Springer-Verlag, New York, 2000.

[M] D. Mumford. Abelian varieties. Second edition. Oxford Univ. Press, Oxford, 1985.

[OSS] C. Okonek, M. Schneider, H. Spindler. Vector Bundles on Complex Projective Spaces. Birkhäuser, 1980.

[PT1] I.Penkov, A. S. Tikhomirov. Rank-2 vector bundles on ind-Grassmannians. In: Algebra, arithmetic, and geometry: in honor of Yu. I. Manin, vol. II, Progress Math., 270, Birkhaeuser, Boston-BaselBerlin 2009, pp. 555-572.

[PT2] I. B. Penkov, A. S. Tikhomirov. Triviality of vector bundles on twisted ind-Grassmannians. Matematicheskij Sbornik 202 (2011) No.1, 65-104 (Russian). English translation: Sbornik: Mathematics 202 (2011) No.1, 61-99.

[PT3] I. B. Penkov, A. S. Tikhomirov. Linear ind-Grassmannnians. Pure and Appl. Math. Quarterly, to appear.

[S1] E. Sato. On the decomposability of infinitely extendable vector bundles on projective spaces and Grassmann varieties. J. Math. Kyoto Univ. 17 (1977), 127-150.

[S2] E. Sato. On infinitely extendable vector bundles on G/P. J. Math. Kyoto Univ. 19 (1979), 171-189.

[S3] E. Sato. The decomposability of an infinitely extendable vector bundle on the projective space, II. Intern. Symp. on Algebraic Geometry, Kyoto, 1977, 663-672.

[T] A. N. Tyurin. Vector bundles of finite rank over infinite varieties. Math. USSR Izvestija 10 (1976), 1187-1204.

Jacobs University Bremen, School of Engineering and Science, Campus Ring 1, 28759 Bremen, GERMANY

E-mail address: i.penkov@jacobs-university.de

Department of Mathematics, State Pedagogical University, Respublikanskaya Str. 108 150000 YaroslaVl, Russia

E-mail address: astikhomirov@mail.ru 